\title{
Electroweak precision observables and Higgs-boson signal strengths in the Standard Model and beyond: present and future
}

J. de Blas, ${ }^{a}$ M. Ciuchini, ${ }^{b}$ E. Franco, ${ }^{a}$ S. Mishima, ${ }^{c}$ M. Pierini, ${ }^{d}$ L. Reina ${ }^{e, f}$ and

L. Silvestrini ${ }^{a}$

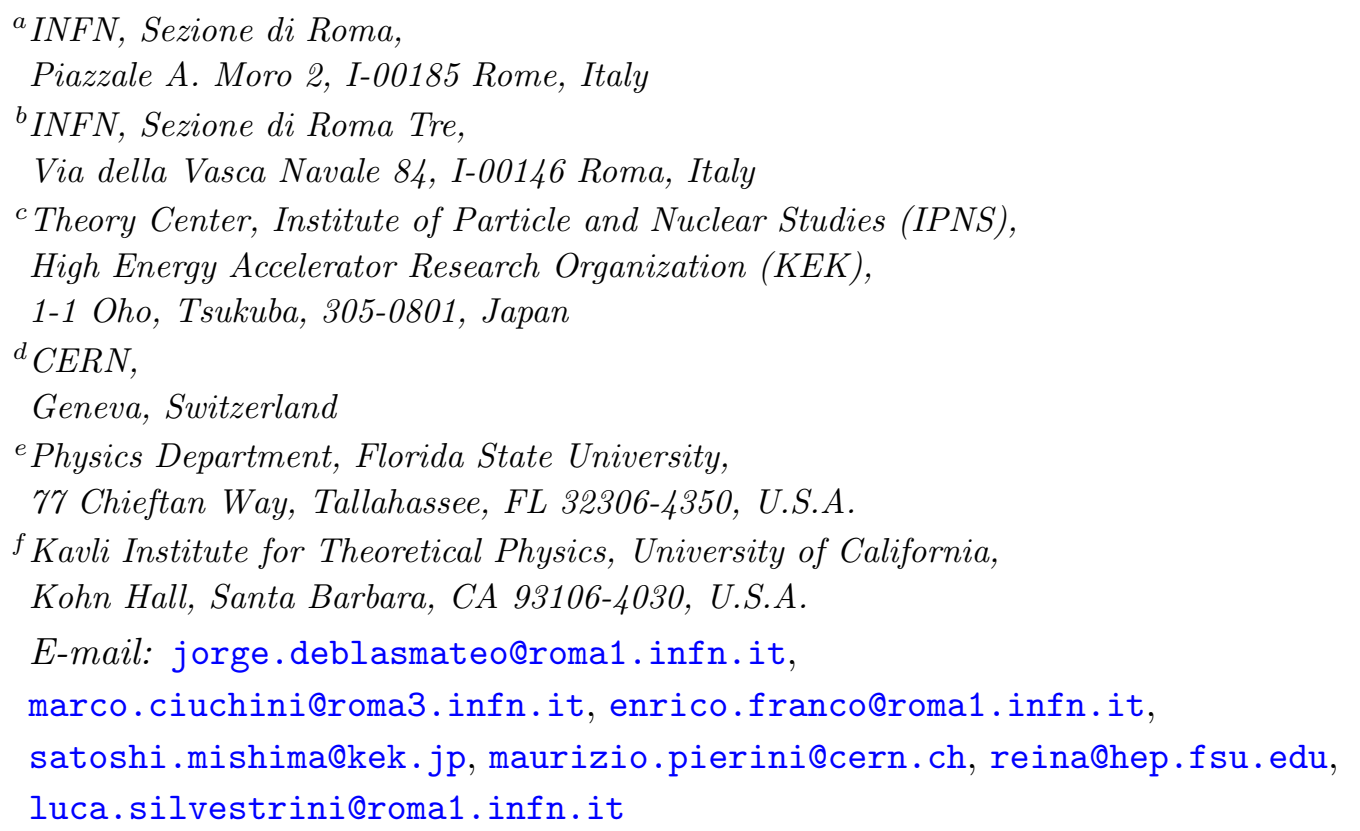

ABSTRACT: We present results from a state-of-the-art fit of electroweak precision observables and Higgs-boson signal-strength measurements performed using 7 and $8 \mathrm{TeV}$ data from the Large Hadron Collider. Based on the HEPfit package, our study updates the traditional fit of electroweak precision observables and extends it to include Higgs-boson measurements. As a result we obtain constraints on new physics corrections to both electroweak observables and Higgs-boson couplings. We present the projected accuracy of the fit taking into account the expected sensitivities at future colliders.

Keywords: Higgs Physics, Beyond Standard Model, Quark Masses and SM Parameters, Perturbative QCD

ARXIV EPRINT: 1608.01509 


\section{Contents}

1 Introduction 1

2 The HEPfit package $\quad 2$

3 Electroweak precision fit in the Standard Model 2

4 Electroweak precision fit beyond the Standard Model $\quad 8$

4.1 Non-standard oblique corrections 8

$\begin{array}{lll}4.2 & \text { Modified } Z b \bar{b} \text { couplings } & 11\end{array}$

$\begin{array}{lll}4.3 & \text { Modified } Z b \bar{b} \text { couplings and oblique corrections } & 11\end{array}$

5 Constraints on Higgs-boson couplings $\quad 12$

$6 \quad$ Expected sensitivities at future lepton colliders 19

$\begin{array}{lll}7 & \text { Conclusions } & 27\end{array}$

\section{Introduction}

Looking for indirect evidence of physics beyond the Standard Model (SM) has become a strong component of the Large Hadron Collider (LHC) physics program. Run I of the LHC revealed the existence of a Higgs boson $(H)$ with characteristics very similar to the Higgs boson of the SM. Identifying the $H$ particle with the SM Higgs boson fully determines the SM Lagrangian, so that all electroweak precision observables (EWPO) and all Higgs-boson couplings can be predicted within the SM. Thus, EWPO and Higgs-boson observables play a key role in constraining extensions of the SM and in searching for new physics (NP).

In this paper we present a global fit of both EWPO and Higgs-boson signal strengths, based on results obtained at LEP, SLC, the Tevatron, and during Run I of the LHC, at both 7 and $8 \mathrm{TeV}$ center-of-mass energies. The fit is carried out using the HEPfit package $[1,2]$, a general tool to combine direct and indirect constraints on the SM and its extensions. In particular, we use HEPfit to perform a statistical analysis of EWPO and Higgs-boson signal-strength measurements in the SM and beyond. Most importantly, we obtain constraints on possible deviations of the Higgs-boson couplings to both gauge bosons and fermions from the SM prediction. Finally, we investigate the impact of the highluminosity upgrade of the LHC and of future $e^{+} e^{-}$colliders on the precision of the fit in the SM and beyond. Our analysis updates the study of ref. [3] and extends it to include recent Higgs-boson physics results. Results from the initial stages of this project were presented in $[4-7]$ and have by now been updated to reflect all the most recent developments in theoretical calculations and experimental measurements. A model-independent study of 
NP effects on both EWPO and Higgs-boson couplings based on an effective-field-theory approach will be presented in a forthcoming paper [8].

Recent updates of global fits to EWPO in the SM and beyond, as well as constraints on Higgs-boson couplings, have been presented in refs. $[9,10]$. In spite of the different statistical methods and of the different inputs, we obtain compatible results for the EWPO fit. We however consider more NP parameterizations, implement constraints from Higgsboson signal strengths, and extend the analysis of future accuracies to more scenarios.

The paper is organized as follows. In section 2 we briefly describe the HEPfit package. In sections 3 and 4 we summarize results for the electroweak (EW) precision fits of the SM and its extensions, while we illustrate in section 5 the constraints we obtain for nonstandard Higgs-boson couplings. The impact of future colliders on our analysis is discussed in section 6 . In section 7 we present our conclusions.

\section{The HEPfit package}

The HEPfit package ${ }^{1}$ is a general tool to combine direct and indirect constraints on the Standard Model and its extensions, available under the GNU General Public License (GPL) [2]. The HEPfit code can be extended to include new observables and NP models which can be added to the main core as external modules. Exploiting the Markov-Chain Monte-Carlo implementation provided in the Bayesian Analysis Toolkit (BAT) [11], HEPfit can be used as a standalone program to perform Bayesian statistical analyses. Alternatively, it can be used in library mode to compute observables in any implemented model, allowing for phenomenological analyses in any statistical framework. The interested reader can find more details on HEPfit in refs. [1,2]. The first application of the HEPfit code has been to update the EW precision fit presented in ref. [5], a detailed explanation of which can be found in [3] and references therein.

In this paper we use HEPfit to perform a Bayesian statistical analysis of EWPO and Higgs-boson observables in the SM and beyond. The code for the EWPO and Higgs observables has been written from scratch. The EWPO results have been successfully validated against ZFITTER [12].

\section{Electroweak precision fit in the Standard Model}

In this section we update the fit of EWPO presented in refs. [3, 5], where all relevant formulæ and a detailed overview of the literature can be found. With respect to ref. [3], we include the full two-loop fermionic EW corrections to $Z$ partial decay widths computed in ref. [25], and the four-loop approximate QCD corrections to the $W$ mass computed in ref. [26-28] (we use the updated semi-analytical formula given in ref. [29]).

Among the input parameters, $G_{\mu}$ and $\alpha$ are fixed $\left(G_{\mu}=1.1663787 \times 10^{-5} \mathrm{GeV}^{-2}\right.$, and $\alpha=1 / 137.035999139$ [10]), while $\alpha_{s}\left(M_{Z}\right), \Delta \alpha_{\text {had }}^{(5)}\left(M_{Z}\right), M_{Z}, m_{t}$, and $m_{H}$ are taken as floating. We use flat priors for all the SM input parameters, and include the information of their

\footnotetext{
${ }^{1}$ Formerly known as SUSYfit, the package has now grown to include more physical observables and multiple models.
} 


\begin{tabular}{|c|c|c|c|c|c|c|}
\hline & Ref. & Measurement & Posterior & Prediction & 1D Pull & nD Pull \\
\hline$\alpha_{s}\left(M_{Z}\right)$ & {$[10]$} & $0.1179 \pm 0.0012$ & $0.1180 \pm 0.0011$ & $0.1185 \pm 0.0028$ & -0.2 & \\
\hline$\Delta \alpha_{\mathrm{had}}^{(5)}\left(M_{Z}\right)$ & {$[13]$} & $0.02750 \pm 0.00033$ & $0.02747 \pm 0.00025$ & $0.02743 \pm 0.00038$ & 0.04 & \\
\hline$M_{Z}[\mathrm{GeV}]$ & {$[14]$} & $91.1875 \pm 0.0021$ & $91.1879 \pm 0.0020$ & $91.199 \pm 0.011$ & -1.0 & \\
\hline$m_{t}[\mathrm{GeV}]$ & {$[15]$} & $173.34 \pm 0.76$ & $173.61 \pm 0.73$ & $176.6 \pm 2.5$ & -1.3 & \\
\hline$m_{H}[\mathrm{GeV}]$ & {$[16]$} & $125.09 \pm 0.24$ & $125.09 \pm 0.24$ & $102.8 \pm 26.3$ & 0.8 & \\
\hline$M_{W}[\mathrm{GeV}]$ & {$[17]$} & $80.385 \pm 0.015$ & $80.3644 \pm 0.0061$ & $80.3604 \pm 0.0066$ & 1.5 & \\
\hline$\Gamma_{W}[\mathrm{GeV}]$ & {$[18]$} & $2.085 \pm 0.042$ & $2.08872 \pm 0.00064$ & $2.08873 \pm 0.00064$ & -0.2 & \\
\hline $\sin ^{2} \theta_{\mathrm{eff}}^{\mathrm{lept}}\left(Q_{\mathrm{FB}}^{\mathrm{had}}\right)$ & {$[14]$} & $0.2324 \pm 0.0012$ & $0.231464 \pm 0.000087$ & $0.231435 \pm 0.000090$ & 0.8 & \\
\hline$P_{\tau}^{\mathrm{pol}}=\mathcal{A}_{\ell}$ & {$[14]$} & $0.1465 \pm 0.0033$ & $0.14748 \pm 0.00068$ & $0.14752 \pm 0.00069$ & -0.4 & \\
\hline$\Gamma_{Z}[\mathrm{GeV}]$ & {$[14]$} & $2.4952 \pm 0.0023$ & $2.49420 \pm 0.00063$ & $2.49405 \pm 0.00068$ & 0.5 & \\
\hline$\sigma_{h}^{0}[\mathrm{nb}]$ & {$[14]$} & $41.540 \pm 0.037$ & $41.4903 \pm 0.0058$ & $41.4912 \pm 0.0062$ & 1.3 & 0.7 \\
\hline$R_{\ell}^{0}$ & {$[14]$} & $20.767 \pm 0.025$ & $20.7485 \pm 0.0070$ & $20.7472 \pm 0.0076$ & 0.8 & \\
\hline$A_{\mathrm{FB}}^{0, \ell}$ & {$[14]$} & $0.0171 \pm 0.0010$ & $0.01631 \pm 0.00015$ & $0.01628 \pm 0.00015$ & 0.8 & \\
\hline $\mathcal{A}_{\ell}(\mathrm{SLD})$ & {$[14]$} & $0.1513 \pm 0.0021$ & $0.14748 \pm 0.00068$ & $0.14765 \pm 0.00076$ & 1.7 & \\
\hline $\mathcal{A}_{c}$ & {$[14]$} & $0.670 \pm 0.027$ & $0.66810 \pm 0.00030$ & $0.66817 \pm 0.00033$ & 0.02 & \\
\hline $\mathcal{A}_{b}$ & {$[14]$} & $0.923 \pm 0.020$ & $0.934650 \pm 0.000058$ & $0.934663 \pm 0.000064$ & -0.6 & \\
\hline$A_{\mathrm{FB}}^{0, c}$ & {$[14]$} & $0.0707 \pm 0.0035$ & $0.07390 \pm 0.00037$ & $0.07399 \pm 0.00042$ & -0.9 & 1.5 \\
\hline$A_{\mathrm{FB}}^{0, b}$ & {$[14]$} & $0.0992 \pm 0.0016$ & $0.10338 \pm 0.00048$ & $0.10350 \pm 0.00054$ & -2.6 & \\
\hline$R_{c}^{0}$ & {$[14]$} & $0.1721 \pm 0.0030$ & $0.172228 \pm 0.000023$ & $0.172229 \pm 0.000023$ & -0.05 & \\
\hline$R_{b}^{0}$ & {$[14]$} & $0.21629 \pm 0.00066$ & $0.215790 \pm 0.000028$ & $0.215788 \pm 0.000028$ & 0.7 & \\
\hline $\sin ^{2} \theta_{\text {eff }}^{e e}$ & [19] & $0.23248 \pm 0.00052$ & & & 2.1 & \\
\hline $\sin ^{2} \theta_{\mathrm{eff}}^{\mu \mu}$ & {$[20]$} & $0.2315 \pm 0.0010$ & & & 0.07 & \\
\hline $\sin ^{2} \theta_{\mathrm{eff}}^{e e}$ & {$[21]$} & $0.23146 \pm 0.00047$ & $0231464+0000087$ & $0231435+0000090$ & 0.1 & \\
\hline $\sin ^{2} \theta_{\mathrm{eff}}^{e e, \mu \mu}$ & {$[22]$} & $0.2308 \pm 0.0012$ & $0.231464 \pm 0.000086$ & $0.231435 \pm 0.000090$ & -0.5 & \\
\hline $\sin ^{2} \theta_{\text {eff }}^{\mu \mu}$ & {$[23]$} & $0.2287 \pm 0.0032$ & & & -0.8 & \\
\hline $\sin ^{2} \theta_{\text {eff }}^{\mu \mu}$ & {$[24]$} & $0.2314 \pm 0.0011$ & & & -0.1 & \\
\hline
\end{tabular}

Table 1. Experimental measurement, result, prediction, and pull for the five input parameters $\left(\alpha_{s}\left(M_{Z}\right), \Delta \alpha_{\text {had }}^{(5)}\left(M_{Z}\right), M_{Z}, m_{t}, m_{H}\right)$, and for the set of EWPO considered in the SM EW fit. The values in the column Prediction are determined without using the corresponding experimental information (see text). Pulls are calculated both as individual pulls (1D Pull) and as global pulls ( $n D$ Pull) for sets of correlated observables (see text), and are given in units of standard deviation. Groups of correlated observables are identified by shades of grey.

experimental measurements in the likelihood. We assume all experimental distributions are Gaussian. Parameters and results for the various EWPO included in the fit are summarized in table 1, where we also give the references from which the measurements have been taken.

With respect to refs. $[3,5]$, we have updated $m_{H}[16]$ and we use the top-quark mass as given by the most up-to-date world average [15]. The values of $M_{Z}[14]$ and $\Delta \alpha_{\text {had }}^{(5)}\left(M_{Z}\right)$ [13] are unchanged. Concerning $\alpha_{s}\left(M_{Z}\right)$, we notice that the most recent PDG average [10] $\left(\alpha_{s}\left(M_{Z}\right)=0.1179 \pm 0.0012\right)$, excluding the result of the EW fit is compatible but sizeably different from the previous one $\left(\alpha_{s}\left(M_{Z}\right)=0.1185 \pm 0.0006\right)$, showing both a lower central value and a larger uncertainty. This is mainly due to the fact that now the uncertainty 
of the combined lattice result for $\alpha_{s}\left(M_{Z}\right)$ is calculated using the same unweighted average procedure as done for the determination of $\alpha_{s}\left(M_{Z}\right)$ in other sub-fields (e.g. hadronic $\tau$ decays, DIS, etc.). A $\chi^{2}$ averaging procedure is then applied to combine the values of $\alpha_{s}\left(M_{Z}\right)$ from the different sub-fields. Previously, the PDG world average for $\alpha_{s}\left(M_{Z}\right)$ had been obtained using a $\chi^{2}$ averaging procedure also to obtain the value of $\alpha_{s}\left(M_{Z}\right)$ from lattice QCD alone [10]. The new procedure turns out to be more conservative and increases the uncertainty on the lattice determination of $\alpha_{s}\left(M_{Z}\right)$ (which was previously dominating the average), leading to a larger final uncertainty of the new world average, and to a reduced fixing power towards the central average value that is now shifted towards lower values induced by measurements in other sub-fields, like the newly added CMS measurement of the $t \bar{t}$ cross section at $\sqrt{s}=7 \mathrm{TeV}$ [30]. Oddly, the new error of the PDG lattice average is comparable to the uncertainty of $\alpha_{s}\left(M_{Z}\right)$ by FLAG [31], although the FLAG error is dominated by an estimate of the uncertainty associated with the truncation of the perturbative series.

In the following, we will use the new PDG average (obtained excluding the EW fit determination) $\alpha_{s}\left(M_{Z}\right)=0.1179 \pm 0.0012$ as a reference value. However, in view of the impact on the EW fit of the increased error, we also present the results for the SM fit with the previous PDG average $\alpha_{s}\left(M_{Z}\right)=0.1185 \pm 0.0005$ to allow the reader to appreciate the effect of the new average. Finally, we have included in the fit the latest determinations of the effective leptonic angle, $\sin ^{2} \theta_{\text {eff }}^{\text {lept }}$, obtained at the Tevatron and at Run I of the LHC.

For each observable, we give the experimental information used as input (Measurement), together with the output of the combined fit (Posterior), and the Prediction of the same quantity. The latter is obtained from the posterior predictive distribution derived from a combined analysis of all the other quantities. The compatibility of the constraints is then tested computing the Pull for each observable as the difference between the corresponding prediction and measurement in units of the combined standard deviation $(1 D$ Pull). Care must be taken in defining the pull for experimentally correlated observables. In this case, we remove from the fit one set of correlated observables at a time and compute the prediction for the set of observables together with their correlation matrix. Adding the experimental covariance matrix to the one obtained from the fit, we compute the log likelihood and the corresponding $p$-value, which we then convert into a global pull for the correlated set of observables assuming Gaussian distributions ( $n D$ Pull).

In figure 1, we show a comparison of the direct measurement (Measurement in table 1), the posterior probability distribution (Posterior in table 1), and the indirect prediction or predictive posterior probability distribution (Prediction in table 1) for the five floating input parameters. These plots show at a glance the impact of the precision of each input parameter on the fit, as well as the agreement between the values preferred by the fit and the direct determinations.

Two of the most important observables in the SM fit are the effective mixing angle, $\sin ^{2} \theta_{\text {eff }}^{\text {lept }}$, and the $W$ mass, $M_{W}$. In figure 2 we show the consistency of the predictions for these observables with the direct experimental measurements, their dependence on the top mass, and the impact of other measurements, such as $m_{H}$ (varied in the range $10 \mathrm{GeV}<m_{H}<1 \mathrm{TeV}$ ) and $\Gamma_{Z}$. 

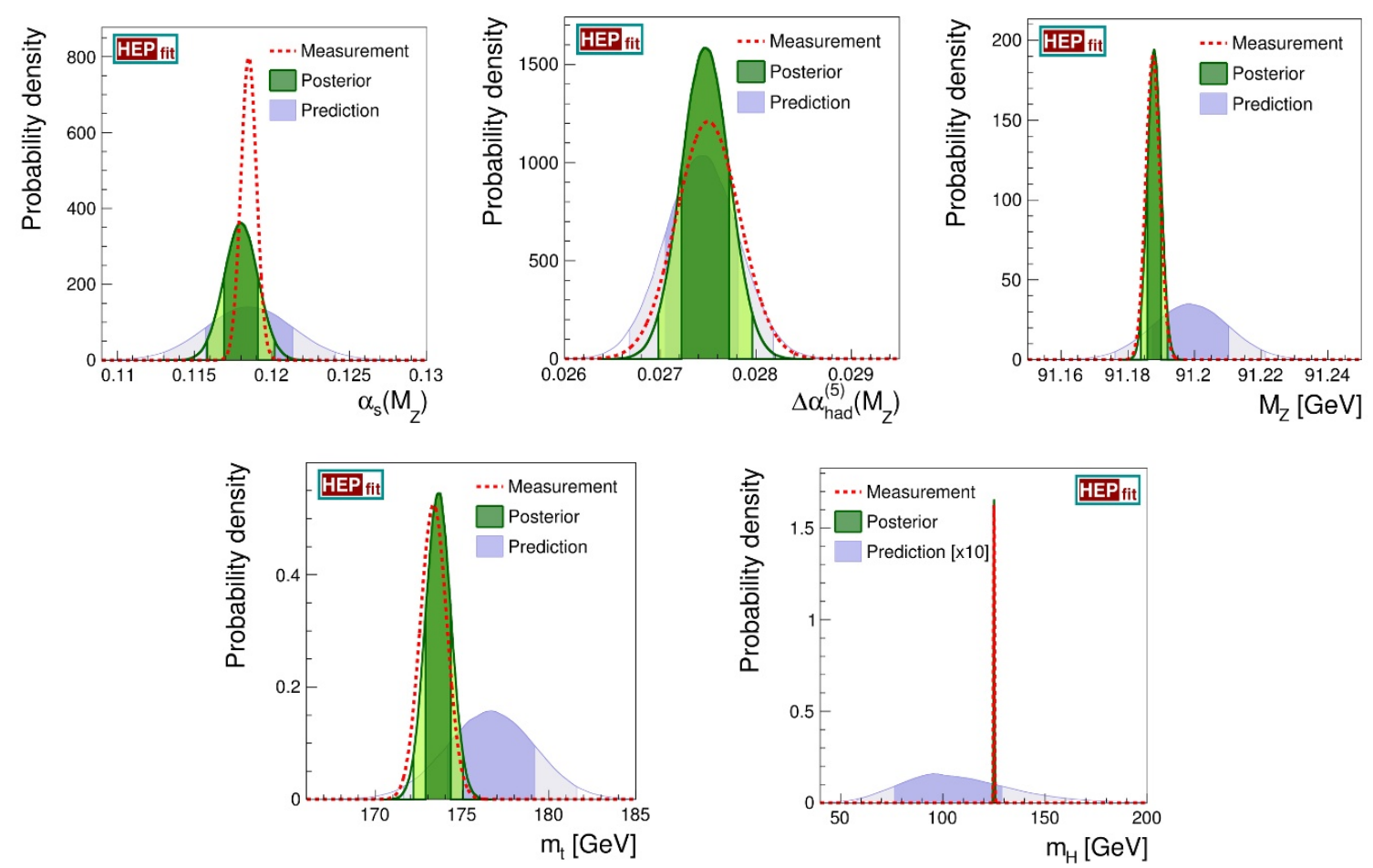

Figure 1. Comparison among the direct measurement, the posterior, and the posterior predictive (or indirect) probability distributions for the input parameters in the SM fit. The latter is obtained from the fit by assuming a flat prior for the parameter under consideration. Dark (light) regions correspond to $68 \%$ (95\%) probability ranges.
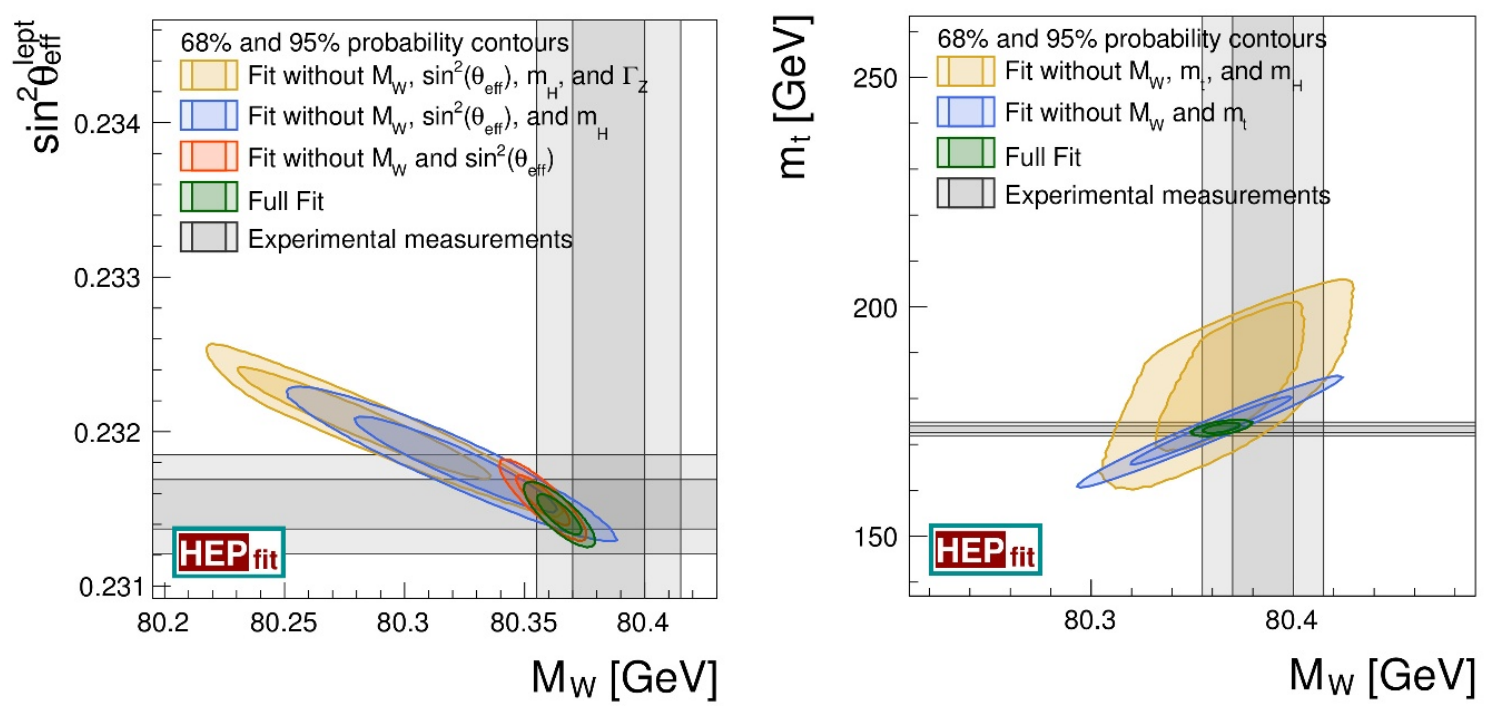

Figure 2. Left: comparison of the indirect constraints on $\sin ^{2} \theta_{\text {eff }}^{\text {lept }}$ and $M_{W}$ with the direct experimental measurements. Dark (light) regions correspond to $68 \%(95 \%)$ probability. Right: the same for $m_{t}$ and $M_{W}$. 


\begin{tabular}{|lcllll|}
\hline & Prediction & \multicolumn{1}{c}{$\alpha_{s}$} & \multicolumn{1}{c}{$\Delta \alpha_{\text {had }}^{(5)}$} & \multicolumn{1}{c|}{$M_{Z}$} & \multicolumn{1}{c|}{$m_{t}$} \\
\hline$M_{W}[\mathrm{GeV}]$ & $80.3618 \pm 0.0080$ & \pm 0.0008 & \pm 0.0060 & \pm 0.0026 & \pm 0.0046 \\
$\Gamma_{W}[\mathrm{GeV}]$ & $2.08849 \pm 0.00079$ & \pm 0.00048 & \pm 0.00047 & \pm 0.00021 & \pm 0.00036 \\
$\Gamma_{Z}[\mathrm{GeV}]$ & $2.49403 \pm 0.00073$ & \pm 0.00059 & \pm 0.00031 & \pm 0.00021 & \pm 0.00017 \\
$\sigma_{h}^{0}[\mathrm{nb}]$ & $41.4910 \pm 0.0062$ & \pm 0.0059 & \pm 0.0005 & \pm 0.0020 & \pm 0.0005 \\
$\sin ^{2} \theta_{\mathrm{eff}}^{\mathrm{lept}}$ & $0.23148 \pm 0.00012$ & \pm 0.00000 & \pm 0.00012 & \pm 0.00002 & \pm 0.00002 \\
$P_{\tau}^{\mathrm{pol}}=\mathcal{A}_{\ell}$ & $0.14731 \pm 0.00093$ & \pm 0.00003 & \pm 0.00091 & \pm 0.00012 & \pm 0.00019 \\
$\mathcal{A}_{c}$ & $0.66802 \pm 0.00041$ & \pm 0.00001 & \pm 0.00040 & \pm 0.00005 & \pm 0.00008 \\
$\mathcal{A}_{b}$ & $0.934643 \pm 0.000076$ & \pm 0.000003 & \pm 0.000075 & \pm 0.000010 & \pm 0.000005 \\
$A_{\mathrm{FB}}^{0, \ell}$ & $0.01627 \pm 0.00021$ & \pm 0.00001 & \pm 0.00020 & \pm 0.00003 & \pm 0.00004 \\
$A_{\mathrm{FB}}^{0, c}$ & $0.07381 \pm 0.00052$ & \pm 0.00002 & \pm 0.00050 & \pm 0.00007 & \pm 0.00010 \\
$A_{\mathrm{FB}}^{0, b}$ & $0.10326 \pm 0.00067$ & \pm 0.00002 & \pm 0.00065 & \pm 0.00008 & \pm 0.00013 \\
$R_{\ell}^{0}$ & $20.7478 \pm 0.0077$ & \pm 0.0074 & \pm 0.0020 & \pm 0.0003 & \pm 0.0003 \\
$R_{c}^{0}$ & $0.172222 \pm 0.000026$ & \pm 0.000023 & \pm 0.000007 & \pm 0.000001 & \pm 0.000009 \\
$R_{b}^{0}$ & $0.215800 \pm 0.000030$ & \pm 0.000013 & \pm 0.000004 & \pm 0.000000 & \pm 0.000026 \\
\hline
\end{tabular}

Table 2. SM predictions computed using the theoretical expressions for the EWPO without the corresponding experimental constraints, and individual uncertainties associated with each input parameter, except for $m_{H}$ (see text).

Looking at the pulls in table 1 , one can notice that there is an overall agreement between EWPO and SM predictions. Only $A_{\mathrm{FB}}^{0, b}$ shows some tension between existing measurements and the result of the SM precision fit. Care must be taken when interpreting this as a possible hint of NP, for deviations at this level $(\sim 2 \sigma)$ are likely to occur when fitting this many observables. Having this in mind, this anomaly will be taken into account in exploring possible parameterizations of NP effects in section 4.

In table 2 we present the full predictions for all EWPO (computed using the theoretical expressions used in the fit without the experimental constraints on the observables) with the breakdown of the parametric uncertainty induced by $1 \sigma$ variations of the input parameters. We do not include in that table the corresponding column for $m_{H}$, since its leading contributions to the EWPO are logarithmic, and hence its error does not induce a significant uncertainty in the predictions. In several cases, the largest contribution to the parametric errors comes from the uncertainty in $\Delta \alpha_{\text {had }}^{(5)}\left(M_{Z}\right)$. This is the dominant source for $\sin ^{2} \theta_{\text {eff }}^{\text {lept }}$ and hence for the different asymmetries. The uncertainties of $M_{W}$ and the pseudo-observables involving decay widths, on the other hand, receive sizeable contributions from several or all input parameters. In particular, with the new PDG value, $\alpha_{s}\left(M_{Z}\right)$ becomes the dominant source of uncertainty in all observables involving the hadronic decay width, with the exception of $R_{b}^{0}$, whose error is controlled by that of $m_{t}$.

For the sake of comparison, we repeat the fit using the old PDG determination of $\alpha_{s}\left(M_{Z}\right)$ and report the results in tables 3 and 4 . The effect is particularly visible in all observables involving the hadronic decay width. 


\begin{tabular}{|c|c|c|c|c|c|c|}
\hline & Ref. & Measurement & Posterior & Prediction & 1D Pull & $\mathrm{nD}$ Pull \\
\hline$\alpha_{s}\left(M_{Z}\right)$ & {$[10]$} & $0.11850 \pm 0.00050$ & $0.11850 \pm 0.00049$ & $0.1186 \pm 0.0028$ & 0.1 & \\
\hline$\Delta \alpha_{\text {had }}^{(5)}\left(M_{Z}\right)$ & {$[13]$} & $0.02750 \pm 0.00033$ & $0.02747 \pm 0.00025$ & $0.02743 \pm 0.00038$ & -0.2 & \\
\hline$M_{Z}[\mathrm{GeV}]$ & {$[14]$} & $91.1875 \pm 0.0021$ & $91.1879 \pm 0.0021$ & $91.198 \pm 0.011$ & -0.9 & \\
\hline$m_{t}[\mathrm{GeV}]$ & {$[15]$} & $173.34 \pm 0.76$ & $173.61 \pm 0.73$ & $176.7 \pm 2.5$ & 1.1 & \\
\hline$m_{H}[\mathrm{GeV}]$ & {$[16]$} & $125.09 \pm 0.24$ & $125.09 \pm 0.24$ & $102.4 \pm 26.4$ & -0.6 & \\
\hline$M_{W}[\mathrm{GeV}]$ & {$[17]$} & $80.385 \pm 0.015$ & $80.3641 \pm 0.0060$ & $80.3601 \pm 0.0066$ & -1.7 & \\
\hline$\Gamma_{W}[\mathrm{GeV}]$ & {$[18]$} & $2.085 \pm 0.042$ & $2.08893 \pm 0.00051$ & $2.08893 \pm 0.00051$ & 0.0 & \\
\hline $\sin ^{2} \theta_{\text {eff }}^{\text {lept }}\left(Q_{\mathrm{FB}}^{\mathrm{had}}\right)$ & {$[14]$} & $0.2324 \pm 0.0012$ & $0.231466 \pm 0.000086$ & $0.231437 \pm 0.000090$ & -0.8 & \\
\hline$P_{\tau}^{\mathrm{pol}}=\mathcal{A}_{\ell}$ & {$[14]$} & $0.1465 \pm 0.0033$ & $0.14746 \pm 0.00068$ & $0.14751 \pm 0.00069$ & 0.1 & \\
\hline$\Gamma_{Z}[\mathrm{GeV}]$ & {$[14]$} & $2.4952 \pm 0.0023$ & $2.49445 \pm 0.00040$ & $2.49439 \pm 0.00041$ & 0.4 & \\
\hline$\sigma_{h}^{0}[\mathrm{nb}]$ & {$[14]$} & $41.540 \pm 0.037$ & $41.4878 \pm 0.0031$ & $41.4880 \pm 0.0032$ & 1.3 & 0.7 \\
\hline$R_{\ell}^{0}$ & {$[14]$} & $20.767 \pm 0.025$ & $20.7516 \pm 0.0034$ & $20.7513 \pm 0.0035$ & 0.6 & \\
\hline$A_{\mathrm{FB}}^{0, \ell}$ & {$[14]$} & $0.0171 \pm 0.0010$ & $0.01631 \pm 0.00015$ & $0.01627 \pm 0.00015$ & 0.9 & \\
\hline $\mathcal{A}_{\ell}(\mathrm{SLD})$ & {$[14]$} & $0.1513 \pm 0.0021$ & $0.14746 \pm 0.00068$ & $0.14762 \pm 0.00076$ & 1.7 & \\
\hline $\mathcal{A}_{c}$ & {$[14]$} & $0.670 \pm 0.027$ & $0.66809 \pm 0.00030$ & $0.66816 \pm 0.00033$ & 0.03 & \\
\hline $\mathcal{A}_{b}$ & {$[14]$} & $0.923 \pm 0.020$ & $0.934648 \pm 0.000058$ & $0.934661 \pm 0.000064$ & -0.4 & \\
\hline$A_{\mathrm{FB}}^{0, c}$ & {$[14]$} & $0.0707 \pm 0.0035$ & $0.07389 \pm 0.00037$ & $0.07398 \pm 0.00042$ & -0.9 & 1.5 \\
\hline$A_{\mathrm{FB}}^{0, b}$ & {$[14]$} & $0.0992 \pm 0.0016$ & $0.10337 \pm 0.00048$ & $0.10348 \pm 0.00054$ & -2.5 & \\
\hline$R_{c}^{0}$ & {$[14]$} & $0.1721 \pm 0.0030$ & $0.172238 \pm 0.000013$ & $0.172239 \pm 0.000013$ & -0.1 & \\
\hline$R_{b}^{0}$ & {$[14]$} & $0.21629 \pm 0.00066$ & $0.215784 \pm 0.000025$ & $0.215783 \pm 0.000026$ & 0.8 & \\
\hline $\sin ^{2} \theta_{\mathrm{eff}}^{e e}$ & {$[19]$} & $0.23248 \pm 0.00053$ & & & 2.1 & \\
\hline $\sin ^{2} \theta_{\text {eff }}^{\mu \mu}$ & {$[20]$} & $0.2315 \pm 0.0010$ & & & 0.1 & \\
\hline $\sin ^{2} \theta_{\text {eff }}^{e e}$ & {$[21]$} & $0.23146 \pm 0.00047$ & $0.231466 \pm 0.000086$ & $0.231437 \pm 0.000090$ & 0.2 & \\
\hline $\sin ^{2} \theta_{\mathrm{eff}}^{e e, \mu \mu}$ & {$[22]$} & $0.2308 \pm 0.0012$ & & & -0.5 & \\
\hline $\sin ^{2} \theta_{\text {eff }}^{\mu \mu}$ & {$[23]$} & $0.2287 \pm 0.0032$ & & & -0.8 & \\
\hline $\sin ^{2} \theta_{\text {eff }}^{\mu \mu}$ & {$[24]$} & $0.2314 \pm 0.0011$ & & & -0.3 & \\
\hline
\end{tabular}

Table 3. Same as table 1 using the old PDG determination of $\alpha_{s}\left(M_{Z}\right)$. 


\begin{tabular}{|lcllll|}
\hline & Prediction & \multicolumn{1}{c}{$\alpha_{s}$} & \multicolumn{1}{c}{$\Delta \alpha_{\text {had }}^{(5)}$} & \multicolumn{1}{c|}{$M_{Z}$} & \multicolumn{1}{c|}{$m_{t}$} \\
\hline$M_{W}[\mathrm{GeV}]$ & $80.3615 \pm 0.0080$ & \pm 0.0003 & \pm 0.0060 & \pm 0.0027 & \pm 0.0046 \\
$\Gamma_{W}[\mathrm{GeV}]$ & $2.08872 \pm 0.00066$ & \pm 0.00020 & \pm 0.00047 & \pm 0.00021 & \pm 0.00036 \\
$\Gamma_{Z}[\mathrm{GeV}]$ & $2.49433 \pm 0.00049$ & \pm 0.00025 & \pm 0.00031 & \pm 0.00021 & \pm 0.00017 \\
$\sigma_{h}^{0}[\mathrm{nb}]$ & $41.4881 \pm 0.0032$ & \pm 0.0024 & \pm 0.0005 & \pm 0.0020 & \pm 0.0005 \\
$\sin ^{2} \theta_{\mathrm{eff}}^{\text {lept }}$ & $0.23149 \pm 0.00012$ & \pm 0.00000 & \pm 0.00012 & \pm 0.00002 & \pm 0.00002 \\
$P_{\tau}^{\text {pol }}=\mathcal{A}_{\ell}$ & $0.14730 \pm 0.00094$ & \pm 0.00001 & \pm 0.00091 & \pm 0.00012 & \pm 0.00019 \\
$\mathcal{A}_{c}$ & $0.66802 \pm 0.00041$ & \pm 0.00001 & \pm 0.00040 & \pm 0.00005 & \pm 0.00008 \\
$\mathcal{A}_{b}$ & $0.934642 \pm 0.000076$ & \pm 0.000001 & \pm 0.000075 & \pm 0.000010 & \pm 0.000005 \\
$A_{\mathrm{FB}}^{0, \ell}$ & $0.01627 \pm 0.00021$ & \pm 0.00000 & \pm 0.00020 & \pm 0.00003 & \pm 0.00004 \\
$A_{\mathrm{FB}}^{0, c}$ & $0.07380 \pm 0.00052$ & \pm 0.00001 & \pm 0.00050 & \pm 0.00007 & \pm 0.00010 \\
$A_{\mathrm{FB}}^{0, b}$ & $0.10325 \pm 0.00067$ & \pm 0.00001 & \pm 0.00065 & \pm 0.00008 & \pm 0.00013 \\
$R_{\ell}^{0}$ & $20.7515 \pm 0.0037$ & \pm 0.0031 & \pm 0.0020 & \pm 0.0003 & \pm 0.0003 \\
$R_{c}^{0}$ & $0.172234 \pm 0.000015$ & \pm 0.000010 & \pm 0.000007 & \pm 0.000001 & \pm 0.000009 \\
$R_{b}^{0}$ & $0.215794 \pm 0.000027$ & \pm 0.000006 & \pm 0.000004 & \pm 0.000000 & \pm 0.000026 \\
\hline
\end{tabular}

Table 4. Same as table 2 using the old PDG determination of $\alpha_{s}\left(M_{Z}\right)$.

\section{Electroweak precision fit beyond the Standard Model}

We now generalize the SM fit considering different sets of parameters which account for NP contributions in several extensions of the SM.

\subsection{Non-standard oblique corrections}

In this section, we use the fit of EWPO to constrain the oblique parameters $S, T, U$ introduced in ref. [32,33] and the $\varepsilon_{1,2,3, b}$ parameters introduced in refs. [34-36].

The $S, T, U$ parameters account for NP effects in the vacuum-polarization amplitudes of the EW gauge bosons and modify all EWPO considered here. The explicit dependence of the EWPO on $S, T$, and $U$ can be found in appendix A of ref. [3] where it was also noticed how the EWPO considered here depend only on the following three specific combinations of the $S, T$, and $U$ parameters (where $s_{W}=\sin \theta_{W}$ and $c_{W}=\cos \theta_{W}$ ),

$$
\begin{aligned}
& A=S-2 c_{W}^{2} T-\frac{\left(c_{W}^{2}-s_{W}^{2}\right) U}{2 s_{W}^{2}}, \\
& B=S-4 c_{W}^{2} s_{W}^{2} T \\
& C=-10\left(3-8 s_{W}^{2}\right) S+\left(63-126 s_{W}^{2}-40 s_{W}^{4}\right) T .
\end{aligned}
$$

Therefore the extracted values of $S, T$, and $U$ are correlated. For this reason, we give in tables 5 and 6 the results of the fit together with the correlation matrix. We also remind the reader that $A$, the only parameter depending on $U$, describes NP contributions to $M_{W}$ and $\Gamma_{W}$, the parameter $C$ describes NP contributions to $\Gamma_{Z}$, and NP contributions to all other 


\begin{tabular}{|c|c|rrr|}
\hline & Result & \multicolumn{3}{|c|}{ Correlation Matrix } \\
\hline$S$ & $0.09 \pm 0.10$ & 1.00 & & \\
$T$ & $0.10 \pm 0.12$ & 0.86 & 1.00 & \\
$U$ & $0.01 \pm 0.09$ & -0.54 & -0.81 & 1.00 \\
\hline
\end{tabular}

Table 5. Results of the fit for the oblique parameters $S, T$, and $U$.

\begin{tabular}{|c|c|lr|}
\hline & Result & \multicolumn{2}{|l|}{ Correlation Matrix } \\
\hline$S$ & $0.10 \pm 0.08$ & 1.00 & \\
$T$ & $0.12 \pm 0.07$ & 0.86 & 1.00 \\
\hline
\end{tabular}

Table 6. Results of the fit for the oblique parameters $S$ and $T$, taking $U=0$.

\begin{tabular}{|c|c|rrrr|}
\hline & Result & \multicolumn{5}{|c|}{ Correlation Matrix } \\
\hline$\delta \varepsilon_{1}$ & $0.0007 \pm 0.0010$ & 1.00 & & & \\
$\delta \varepsilon_{2}$ & $-0.0002 \pm 0.0008$ & 0.82 & 1.00 & & \\
$\delta \varepsilon_{3}$ & $0.0007 \pm 0.0009$ & 0.87 & 0.56 & 1.00 & \\
$\delta \varepsilon_{b}$ & $0.0004 \pm 0.0013$ & -0.34 & -0.32 & -0.24 & 1.00 \\
\hline
\end{tabular}

Table 7. Results of the fit for the $\delta \varepsilon_{i}$ parameters $(i=1,2,3, b)$.

EWPO are proportional to $B$. As illustrated in figure $3, S, T$, and $U$ are compatible with zero, implying the absence of sizeable oblique corrections beyond those predicted by the SM. In general, in models of new physics with linearly realized electroweak symmetry breaking $U$ is largely suppressed relative to $S$ and $T$. For this reason we also specify our fit to the case in which $U=0$ and give the corresponding results in table 6 , and in the bottom plots of figure 3. The results of table 9 later in this section are are also given in the $U=0$ scenario.

Next we consider the $\varepsilon_{1,2,3, b}$ parameters introduced in refs. [34-36]. Unlike the $S, T$, and $U$ parameters discussed above, the $\varepsilon_{i}$ parameters involve SM contributions associated with the top quark and the Higgs boson, SM flavour non-universal vertex corrections, and further vacuum-polarization corrections [37]. Since the SM is now fully known and there is no need to disentangle top-quark and Higgs-boson contributions anymore, we separate the genuine NP contribution from the SM one by introducing $\delta \varepsilon_{i}=\varepsilon_{i}-\varepsilon_{i, \mathrm{SM}}$ for $i=1,2,3, b$, where $\varepsilon_{i}$ are the original parameters and $\varepsilon_{i, \mathrm{SM}}$ contain the SM contribution only. The expressions of the EWPO in terms of $\delta \varepsilon_{i}$ can be found in ref. $[3,5]$.

The results of our fit for the $\delta \varepsilon_{i}$ parameters are summarized in table 7 . Some twodimensional probability distributions are plotted in figure 4. All results are consistent with the SM. Note that, as mentioned above, the $\delta \varepsilon_{i}$ parameters include oblique corrections 

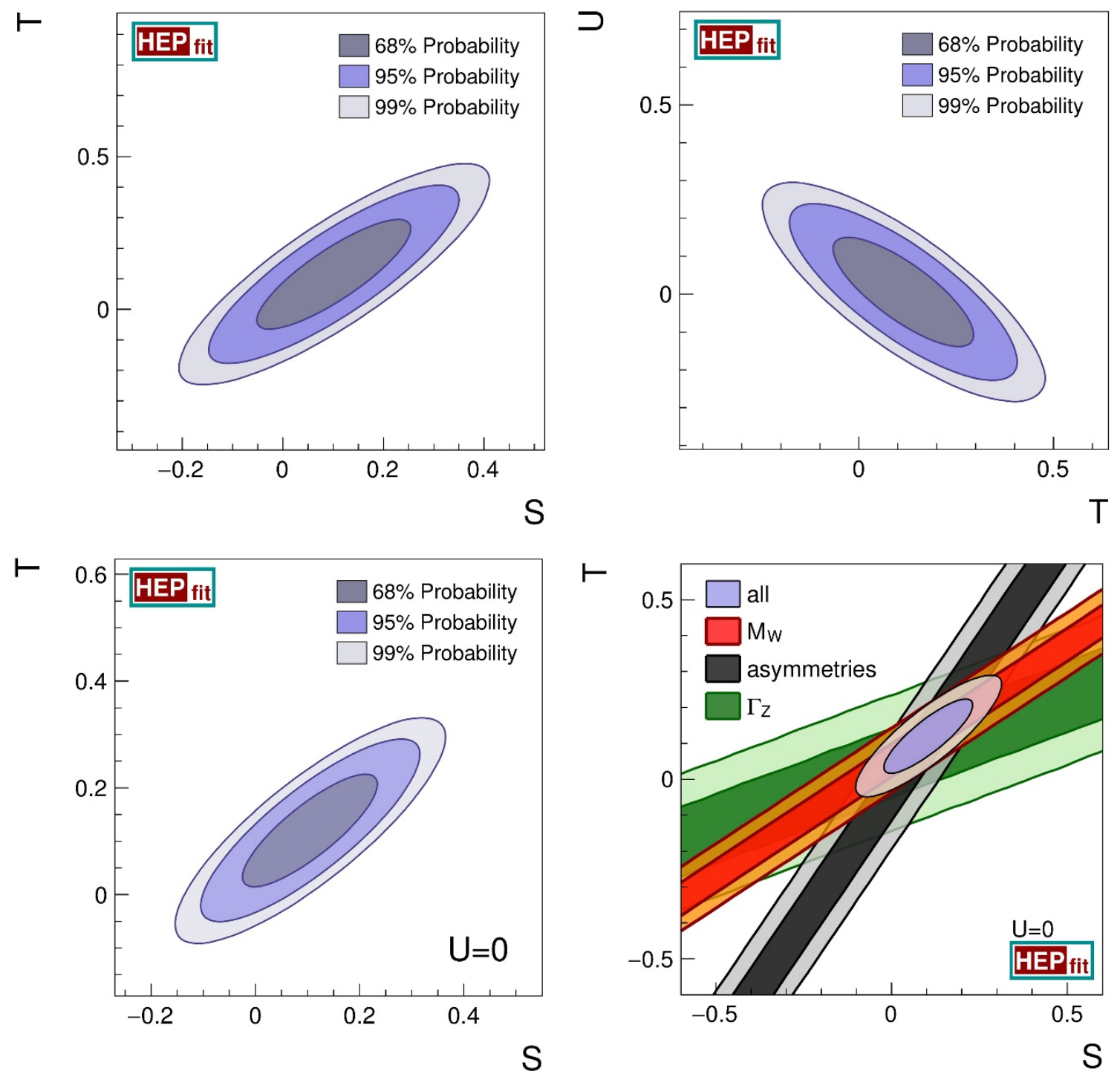

Figure 3. Two-dimensional probability distributions for the oblique parameters $S$ and $T$ (upperleft panel), and $T$ and $U$ (upper-right panel). From darker to lighter the different regions correspond respectively to $68 \%, 95 \%$, and $99 \%$ probability. In the lower panel we show the two-dimensional distributions for $S$ and $T$ fixing $U=0$, together with the individual constraints from $M_{W}$, the asymmetry parameters $\sin ^{2} \theta_{\mathrm{eff}}^{\text {lept }}, P_{\tau}^{\text {pol }}, \mathcal{A}_{f}$, and $A_{\mathrm{FB}}^{0, f}$ with $f=\ell, c, b$, and $\Gamma_{Z}$. In this last plot the dark (light) region corresponds to $68 \%(95 \%)$ probability.

beyond those connected to the $S, T$, and $U$ parameters. More precisely,

$$
\begin{aligned}
& \delta \varepsilon_{1}=\alpha T-W+2 X \frac{\sin \theta_{W}}{\cos \theta_{W}}-Y \frac{\sin ^{2} \theta_{W}}{\cos ^{2} \theta_{W}}, \\
& \delta \varepsilon_{2}=-\frac{\alpha}{4 \sin ^{2} \theta_{W}} U-W+2 X \frac{\sin \theta_{W}}{\cos \theta_{W}}-V \\
& \delta \varepsilon_{3}=\frac{\alpha}{4 \sin ^{2} \theta_{W}} S-W+\frac{X}{\sin \theta_{W} \cos \theta_{W}}-Y,
\end{aligned}
$$



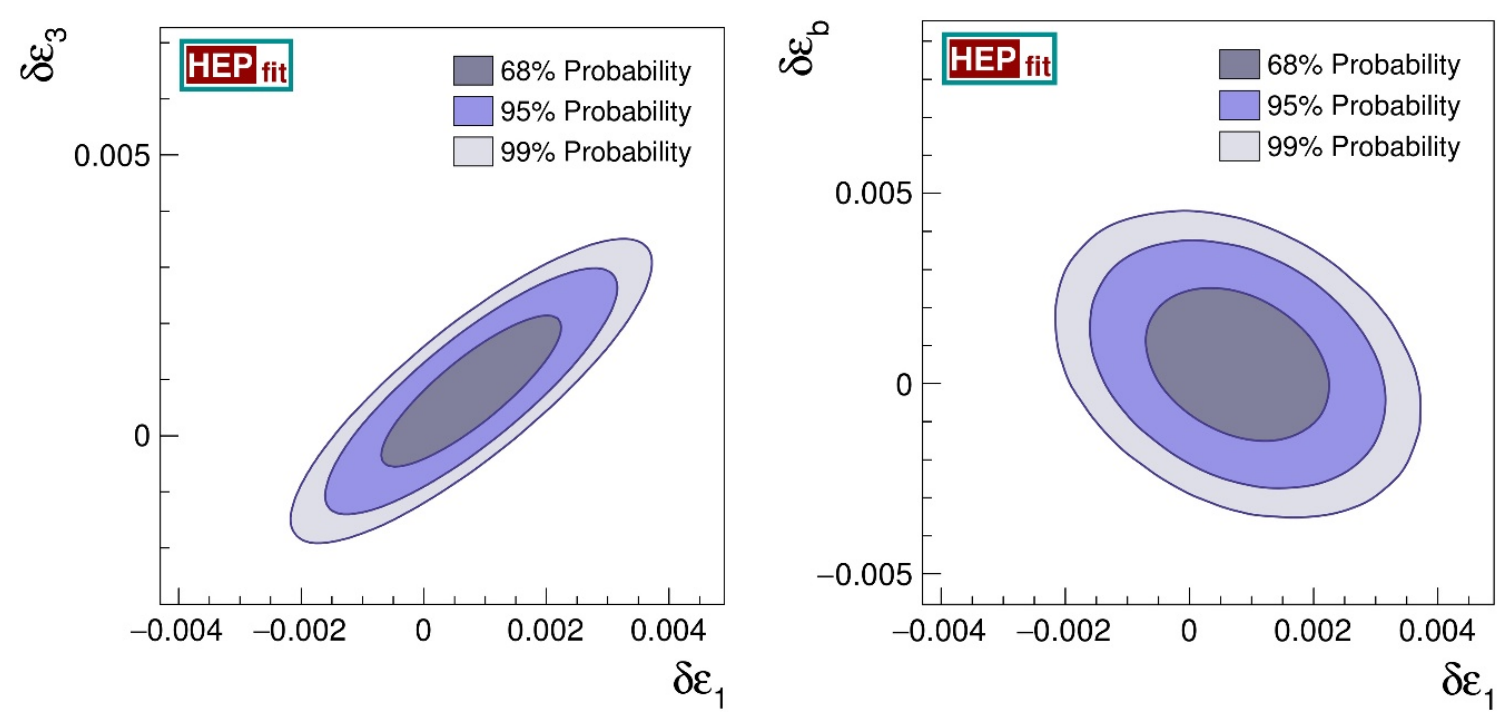

Figure 4. Two-dimensional probability distributions for $\delta \varepsilon_{1}$ and $\delta \varepsilon_{3}$ (left), and $\delta \varepsilon_{1}$ and $\delta \varepsilon_{b}$ (right) varying all $\delta \varepsilon_{i}$ parameters. From darker to lighter the different regions correspond to $68 \%, 95 \%$, and $99 \%$ probability.

where $V, W, X, Y$ are part of the extended set of oblique parameters defined in [37]. With the results in table 7 and the above equations, one can therefore obtain approximate constraints on NP scenarios with vanishing contributions to $S, T$, and/or $U$ but non-zero values of some of the other parameters $(V, W, X$, and $Y)$.

\subsection{Modified $Z b \bar{b}$ couplings}

Motivated by the apparent discrepancy between the SM prediction for $A_{F B}^{0, b}$ and the corresponding experimental result, we also consider here the case where dominant NP contributions appear in the $Z b \bar{b}$ couplings. We parameterize NP contributions to the $Z b \bar{b}$ couplings as follows:

$$
g_{i}^{b}=g_{i, \mathrm{SM}}^{b}+\delta g_{i}^{b} \quad \text { for } i=L, R \text { or } V, A,
$$

and we present results for both $V, A$, and $L, R$ couplings. Details on the definitions of these couplings can be found in ref. [3]. The EW precision fit finds four solutions for these couplings, but two of them are disfavoured by the off-peak measurement of the forwardbackward asymmetry in $e^{+} e^{-} \rightarrow b \bar{b}$ [38]. In table 8 and figure 5 , we present only the solution closer to the SM. The observed deviations from zero of the parameters $\delta g_{i}^{b}$ reflect the deviation from the SM of the measured value of $A_{\mathrm{FB}}^{0, b}$. While the agreement between the SM and $R_{b}^{0}$ results in a preferred value of $\delta g_{L}^{b}$ consistent with the SM at the $2 \sigma$ level, a sizeable contribution to $\delta g_{R}^{b}$ is required to explain the $A_{\mathrm{FB}}^{0, b}$, and the resulting $95 \%$ probability region in the $\delta g_{L}^{b}-\delta g_{R}^{b}$ plane is only marginally compatible with the SM predictions.

\subsection{Modified $Z \boldsymbol{Z} b \bar{b}$ couplings and oblique corrections}

In several extensions of the SM, oblique corrections and modifications of the $Z b \bar{b}$ vertex occur simultaneously, possibly affecting only a specific chirality of the vertex (see for example 


\begin{tabular}{|c|c|cc|}
\hline & Result & \multicolumn{2}{|c|}{ Correlation Matrix } \\
\hline$\delta g_{R}^{b}$ & $0.016 \pm 0.006$ & 1.00 & \\
$\delta g_{L}^{b}$ & $0.002 \pm 0.001$ & 0.90 & 1.00 \\
\hline & & & \\
\hline$\delta g_{V}^{b}$ & $0.018 \pm 0.007$ & 1.00 & \\
$\delta g_{A}^{b}$ & $-0.013 \pm 0.005$ & -0.98 & 1.00 \\
\hline
\end{tabular}

Table 8. Results of the fit for the shifts in the $Z b \bar{b}$ couplings.
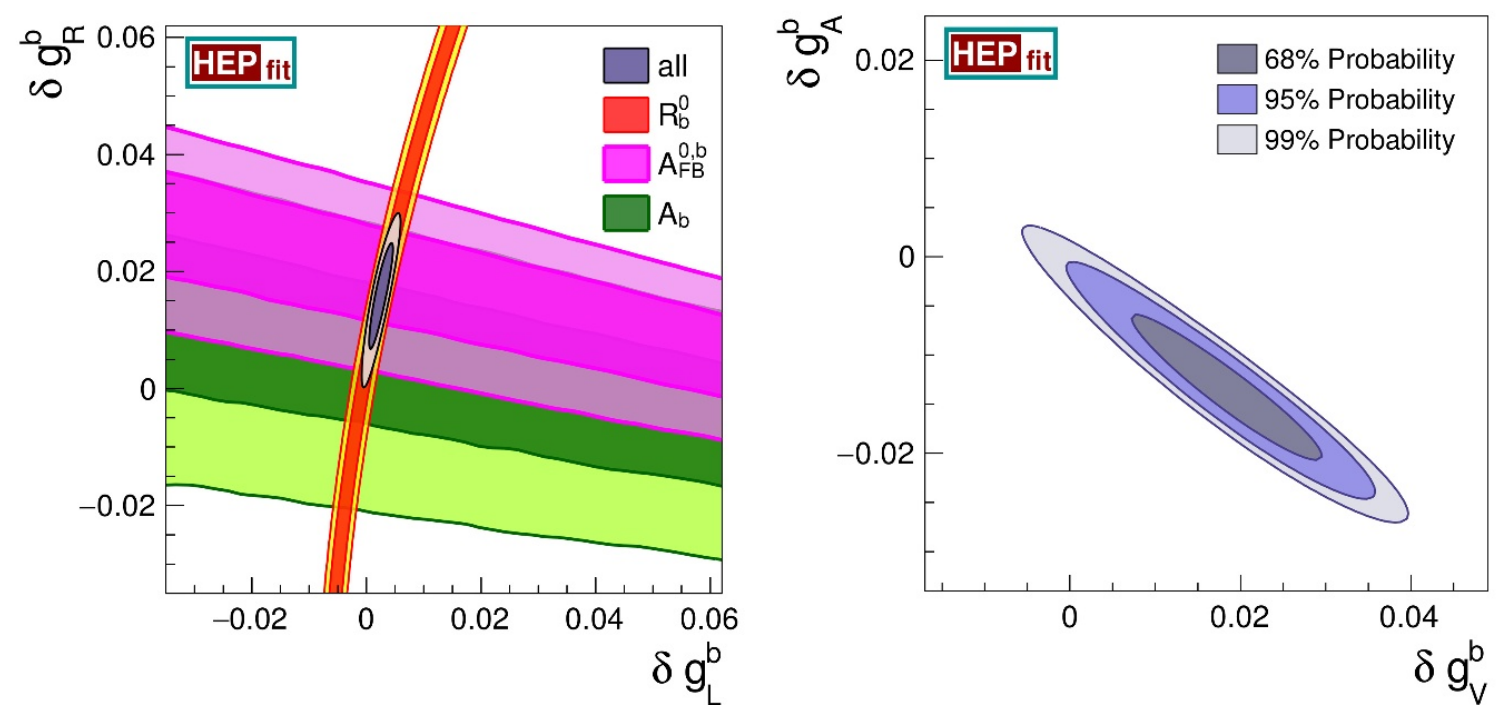

Figure 5. Two-dimensional probability distributions for $\delta g_{R}^{b}, \delta g_{L}^{b}$ (left), and $\delta g_{V}^{b}, \delta g_{A}^{b}$ (right). In the left plot, the dark (light) regions correspond to $68 \%$ (95\%) probability regions.

refs. $[39,40])$. We therefore consider the following cases: oblique contributions with i) $\delta g_{L}^{b}$ and $\delta g_{R}^{b}$, ii) $\delta g_{L}^{b}$ only and iii) $\delta g_{R}^{b}$ only. The corresponding results are presented in table 9 .

\section{Constraints on Higgs-boson couplings}

In addition to the standard set of EWPO, we have considered all most recent measurements of Higgs-boson signal strengths, i.e. the ratio between the measured effective cross section and the corresponding SM prediction $\left(\mu \equiv \sigma / \sigma_{\mathrm{SM}}\right)$, taken from refs. [41, 42] for $H \rightarrow \gamma \gamma$, refs. [43, 44] for $H \rightarrow \tau^{+} \tau^{-}$, refs. [45-47] for $H \rightarrow Z Z$, refs. [48-50] for $H \rightarrow W^{+} W^{-}$, and refs. [51-54] as well as the Tevatron papers $[55,56]$ for $H \rightarrow b \bar{b}$. The Higgs-boson signal strength $\mu$ of a specific Higgs-search analysis can be calculated as

$$
\mu=\sum_{i} w_{i} r_{i} \quad \text { with } \quad r_{i}=\frac{(\sigma \times B r)_{i}}{\left(\sigma_{\mathrm{SM}} \times B r_{\mathrm{SM}}\right)_{i}} \quad \text { and } \quad w_{i}=\frac{\epsilon_{i}\left(\sigma_{\mathrm{SM}} \times B r_{\mathrm{SM}}\right)_{i}}{\sum_{j} \epsilon_{j}\left(\sigma_{\mathrm{SM}} \times B r_{\mathrm{SM}}\right)_{j}}
$$

where $\epsilon_{i}$ are the experimental efficiencies, and the sums run over all channels which can contribute to the final state of the specific analysis. The SM Higgs-boson production cross 


\begin{tabular}{|c|c|rrrr|}
\hline & Result & \multicolumn{3}{|c|}{ Correlation Matrix } \\
\hline$S$ & $0.04 \pm 0.09$ & 1.00 & & & \\
$T$ & $0.08 \pm 0.07$ & 0.86 & 1.00 & & \\
$\delta g_{L}^{b}$ & $0.003 \pm 0.001$ & -0.24 & -0.15 & 1.00 & \\
$\delta g_{R}^{b}$ & $0.017 \pm 0.008$ & -0.29 & -0.22 & 0.91 & 1.00 \\
\hline \multicolumn{5}{|c|}{$\delta g_{R}^{b}=0$} \\
\hline$S$ & $0.10 \pm 0.09$ & 1.00 & & \\
$T$ & $0.12 \pm 0.07$ & 0.85 & 1.00 & \\
$\delta g_{L}^{b}$ & $-0.0001 \pm 0.0006$ & 0.07 & 0.13 & 1.00 \\
\hline \multicolumn{7}{|c|}{$\delta g_{L}^{b}=0$} \\
\hline$S$ & $0.08 \pm 0.09$ & 1.00 & & \\
$T$ & $0.10 \pm 0.07$ & 0.86 & 1.00 & \\
$\delta g_{R}^{b}$ & $0.004 \pm 0.003$ & -0.19 & -0.21 & 1.00 \\
\hline
\end{tabular}

Table 9. Results of the combined fit of the oblique parameters $S$ and $T$, and of the modified $Z b \bar{b}$ couplings, in the case when both $\delta g_{R}^{b}$ and $\delta g_{L}^{b}$ are non zero, and in the case in which either $\delta g_{R}^{b}=0$ or $\delta g_{L}^{b}=0$.

sections (including QCD and, when available, EW corrections) are taken from ref. [57] and the SM Higgs-boson decay rates are taken from ref. [58].

In this section, we specialize our discussion to a minimal NP scenario consisting of an effective theory with only one Higgs boson below the cutoff scale $\Lambda$. Following ref. [59], we assume that custodial symmetry is approximately realized, and that the NP scale is sufficiently large compared to the energies we are testing, so we can truncate the effective Lagrangian at the 2-derivative level. We also assume that gauge fields couple to the NP sector via weak gauging, in which case the coefficients of operators involving field strengths are loop suppressed and we can neglect them. Finally, we assume that fermions are only coupled to the NP via proto-Yukawa interactions, and we take all corrections from NP to be flavor diagonal and universal. This scenario can be described by a general effective Lagrangian of the form (see e.g. [59-62]):

$$
\mathcal{L}_{\text {eff }}=\frac{v^{2}}{4} \operatorname{tr}\left(D_{\mu} \Sigma^{\dagger} D^{\mu} \Sigma\right)\left(1+2 \kappa_{V} \frac{H}{v}+\cdots\right)-m_{i} \bar{f}_{L}^{i}\left(1+2 \kappa_{f} \frac{H}{v}+\cdots\right) f_{R}^{i}+\cdots,
$$

where $v$ is the vacuum expectation value of the Higgs field, and the longitudinal components of the $W$ and $Z$ bosons, $\chi^{a}(x)$, are described by the two-by-two matrix $\Sigma(x)=$ $\exp \left(i \tau^{a} \chi^{a}(x) / v\right)$, with $\tau^{a}$ being the Pauli matrices. The deviations in the Higgs-boson couplings to weak gauge-bosons, $H V V\left(V=Z, W^{ \pm}\right)$, and to fermions, $H f \bar{f}$, are parameterized by the scale factors $\kappa_{V}$ and $\kappa_{f}$ respectively, defined as the ratio between the total Higgs-boson couplings, including NP effects, and the corresponding couplings in the SM (such that $\kappa_{V}=\kappa_{f}=1$ in the SM). We only consider the modification of couplings already existing in the SM and, for loop-induced couplings $(H g g, H \gamma \gamma$, and $H Z \gamma)$, we do not 


\begin{tabular}{|c|cc|}
\hline & Result & $95 \%$ Prob. \\
\hline$\kappa_{V}$ & $1.02 \pm 0.02$ & {$[0.98,1.07]$} \\
\hline
\end{tabular}

Table 10. Results of the fit for the scale factor $\kappa_{V}$ at $68 \%$ and $95 \%$ probabilities.

assume NP contributions in loops. ${ }^{2}$ This class of models is not fully general but it is more directly constrained by the experimental measurements of Higgs-boson couplings. It is also the scenario assumed in both ATLAS and CMS studies of Higgs-boson couplings and allows us to directly compare to their results, giving us the possibility to test both the HEPfit framework and our correct use of the experimental data for Higgs-boson signal-strengths. For a detailed description of the relations between scale factors and the Higgs-boson signal strengths we refer the reader to ref. [57].

In this context we first perform a fit of the EWPO with the only addition of the scale factor $\kappa_{V}$. The only corrections to EWPO are then given by the following 1-loop contributions to the oblique $S$ and $T$ parameters $^{3}[63]$ :

$$
S=\frac{1}{12 \pi}\left(1-\kappa_{V}^{2}\right) \ln \left(\frac{\Lambda^{2}}{m_{H}^{2}}\right), \quad T=-\frac{3}{16 \pi c_{W}^{2}}\left(1-\kappa_{V}^{2}\right) \ln \left(\frac{\Lambda^{2}}{m_{H}^{2}}\right),
$$

where we set the cutoff of the effective Lagrangian to the scale of violation of perturbative unitarity in $W W$ scattering, i.e. $\Lambda=4 \pi v / \sqrt{\left|1-\kappa_{V}^{2}\right|}$. We present the results of the fit for $\kappa_{V}$ in table 10 and figure 6.

The lower bound on $\kappa_{V}$ at $95 \%$ corresponds to a cutoff scale $\Lambda=13 \mathrm{TeV}$ if $\kappa_{V}$ is assumed to be smaller than $1, \Lambda=8.7 \mathrm{TeV}$ if $\kappa_{V}$ is assumed to be larger than 1 , and $\Lambda=8.8 \mathrm{TeV}$ marginalizing over the sign of $1-\kappa_{V}$. The fit disfavours values of $\kappa_{V}<1$ ( $10 \%$ probability), expected for example in composite Higgs models. This problem can be alleviated by adding extra contributions to the oblique parameters [64-67].

The two-dimensional probability distributions for $\kappa_{V}$ and $\kappa_{f}$ obtained from the fit to Higgs-boson signal strengths are summarized in table 11 and shown in figure 7 . The left panel of of figure 7 shows the $95 \%$ probability contours obtained from a fit including only each individual channel (e.g. $H \rightarrow \gamma \gamma$ ), as well as the result from the global fit. Since both production cross sections and decay rates depend on the modified couplings via products of the form $\kappa_{i} \kappa_{j}$, theoretical predictions are symmetric under the simultaneous exchange $\left\{\kappa_{V}, \kappa_{f}\right\} \leftrightarrow\left\{-\kappa_{V},-\kappa_{f}\right\}$. We therefore restrict the parameter space to positive $\kappa_{V}$ only.

\footnotetext{
${ }^{2}$ We notice that, in the presence of NP, the relative experimental efficiencies, $\epsilon_{i}$, will in general be different from their values in the SM. In particular, the appearance of new tensor structures in the vertices could modify the kinematic distributions of the final-state particles, thereby changing the efficiencies. However, since in this work we only consider rescalings of the SM Higgs-boson couplings, we will use SM efficiencies $\epsilon_{i}^{\mathrm{SM}}$ (and hence weight factors $w_{i}^{\mathrm{SM}}$ ) throughout.

${ }^{3}$ Even if we assume that custodial symmetry is preserved by the new interactions, there is still a nonzero contribution to the $T$ parameter. Note that custodial symmetry breaking is actually parameterized by $\Delta \rho=\alpha T$, which, in this scenario, is proportional to the square of $\mathrm{U}(1)_{Y}$ gauge coupling, $g^{\prime}$. Indeed $g^{\prime}$ is one of the parameters that breaks custodial symmetry in the SM, and the new effective interactions only modify the way custodial symmetry is broken. In the limit of $g^{\prime} \rightarrow 0$ there is no contribution to $T$, consistently with the assumption of custodial symmetry in the new physics.
} 


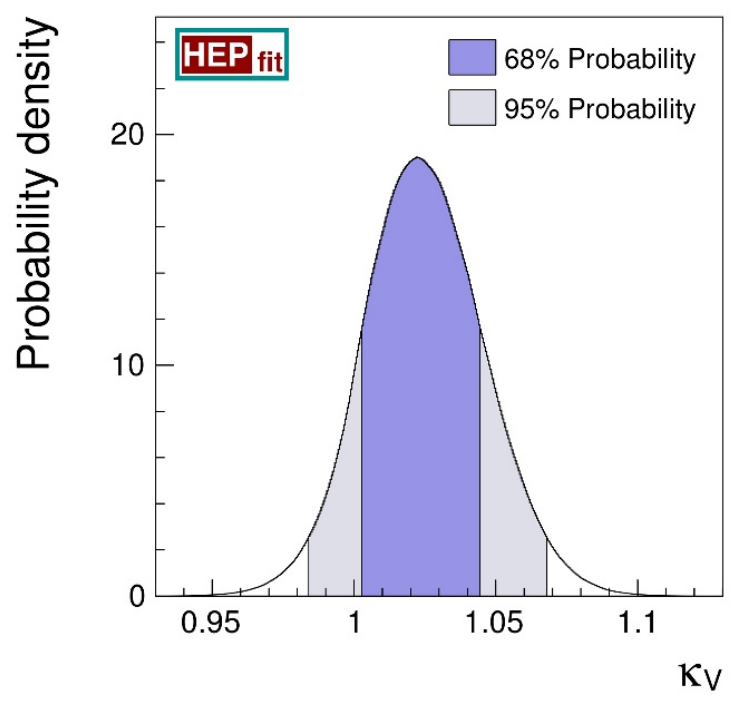

Figure 6. Probability distribution for $\kappa_{V}$ derived from precision EW measurements. The dark and light regions correspond respectively to $68 \%$ and $95 \%$ probabilities.

\begin{tabular}{|c|c|c|lr|}
\hline & Result & $95 \%$ Prob. & Correlation Matrix \\
\hline$\kappa_{V}$ & $1.01 \pm 0.04$ & {$[0.93,1.10]$} & 1.00 & \\
$\kappa_{f}$ & $1.03 \pm 0.10$ & {$[0.83,1.23]$} & 0.31 & 1.00 \\
\hline
\end{tabular}

Table 11. SM-like solution in the fit of $\kappa_{V}$ and $\kappa_{f}$ to the Higgs-boson signal strengths.

\begin{tabular}{|c|c|c|lr|}
\hline & Result & $95 \%$ Prob. & \multicolumn{2}{|l|}{ Correlation Matrix } \\
\hline$\kappa_{V}$ & $1.02 \pm 0.02$ & {$[0.99,1.06]$} & 1.00 & \\
$\kappa_{f}$ & $1.03 \pm 0.10$ & {$[0.85,1.23]$} & 0.14 & 1.00 \\
\hline
\end{tabular}

Table 12. Same as table 11 but considering both the Higgs-boson signal strengths and the EWPO.

Note also that, when performing the global fit to all channels, the region with negative $\kappa_{f}$ is not populated even at $99 \%$ probability, so that we only show positive values of $\kappa_{f}$ in the right-hand-side plot of figure 7. The effect of performing a combined fit of both Higgs-boson signal strengths and EWPO is summarized in table 12 and illustrated in figure 8 (note that in tables 11 and 12 we only show the results corresponding to the SM-like solution, i.e. $\left.\kappa_{V, f}>0\right)$. It is interesting to notice that the constraint on $\kappa_{V}$ from EWPO is stronger than the one obtained from the Higgs-boson signal strengths alone.

We then lift the assumption of custodial symmetry and rescale the $H Z Z$ and $H W^{+} W^{-}$ couplings independently, introducing two parameters $\kappa_{Z}$ and $\kappa_{W}$, while keeping a unique rescaling factor for all fermionic couplings, $\kappa_{f}$. We obtain the results summarized in table 13 and the corresponding probability distributions shown in figure 9, which are consistent with custodial symmetry. We notice that theoretical predictions are symmetric under 

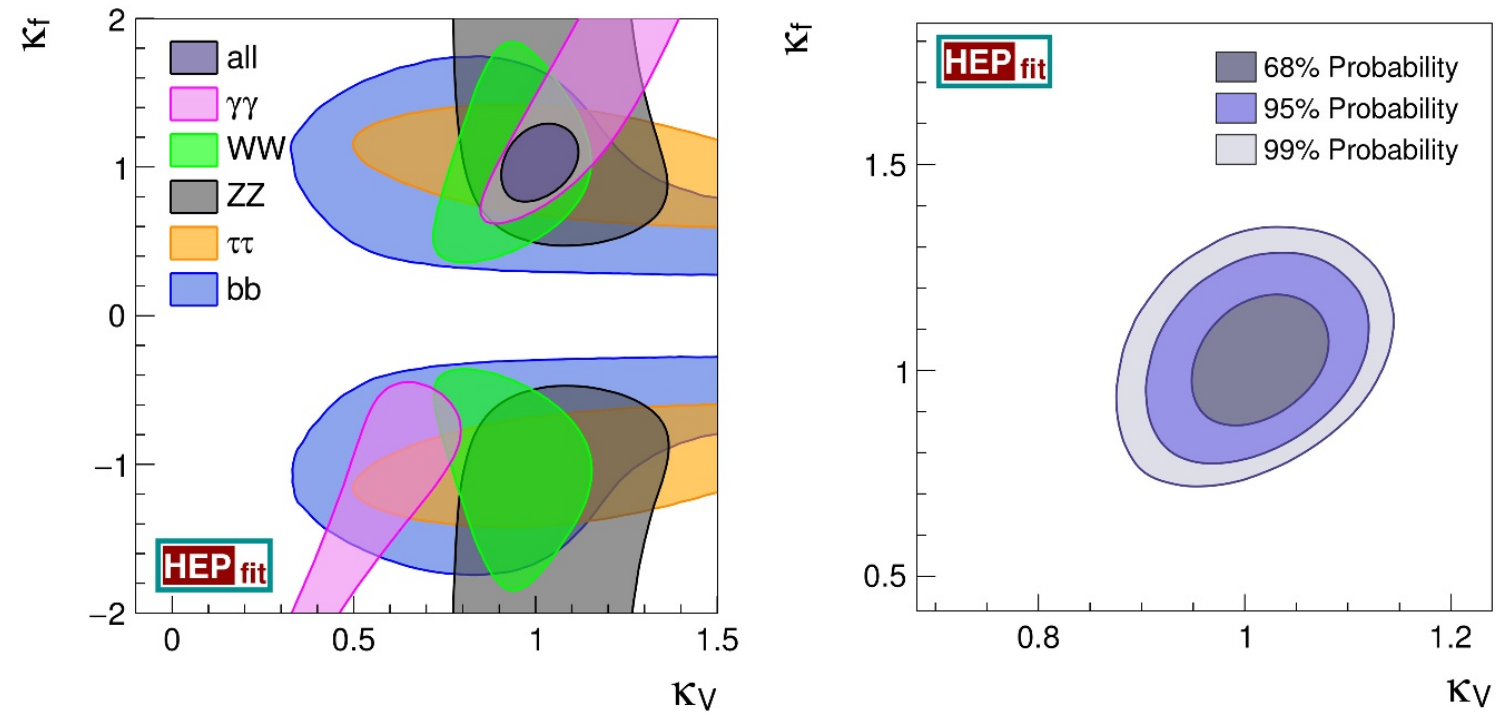

Figure 7. Left: constraints from individual channels at $95 \%$ probability. Right: two-dimensional probability distributions for $\kappa_{V}$ and $\kappa_{f}$ at $68 \%, 95 \%$, and $99 \%$ (darker to lighter), obtained from the fit to the Higgs-boson signal strengths.

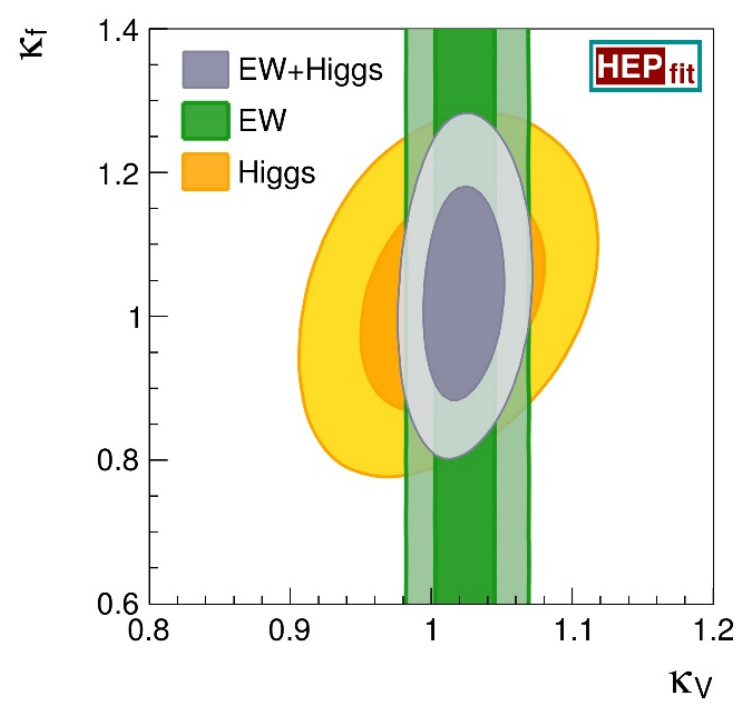

Figure 8. Two-dimensional $68 \%$ (dark) and $95 \%$ (light) probability contours for $\kappa_{V}$ and $\kappa_{f}$ (from darker to lighter), obtained from the fit to the Higgs-boson signal strengths and the EWPO.

the exchanges $\left\{\kappa_{W}, \kappa_{f}\right\} \leftrightarrow\left\{-\kappa_{W},-\kappa_{f}\right\}$ and/or $\kappa_{Z} \leftrightarrow-\kappa_{Z}$, where $\kappa_{Z}$ can flip the sign independent of $\kappa_{W}$, since the interference between the $W$ and $Z$ contributions to the vector-boson fusion cross section is negligible. Hence we have considered only the parameter space where both $\kappa_{W}$ and $\kappa_{Z}$ are positive. In this case, we ignore EWPO in the fit, since setting $\kappa_{W} \neq \kappa_{Z}$ generates power divergences in the oblique corrections, indicating that the detailed information on the UV theory is necessary for calculating the oblique corrections. 


\begin{tabular}{|c|c|c|rrr|}
\hline & Result & $95 \%$ Prob. & Correlation Matrix \\
\hline$\kappa_{W}$ & $1.00 \pm 0.05$ & {$[0.89,1.10]$} & 1.00 & & \\
$\kappa_{Z}$ & $1.07 \pm 0.11$ & {$[0.85,1.27]$} & -0.17 & 1.00 & \\
$\kappa_{f}$ & $1.01 \pm 0.11$ & {$[0.80,1.22]$} & 0.41 & -0.14 & 1.00 \\
\hline
\end{tabular}

Table 13. SM-like solution in the fit of $\kappa_{W}, \kappa_{Z}$, and $\kappa_{f}$ to the Higgs-boson signal strengths.
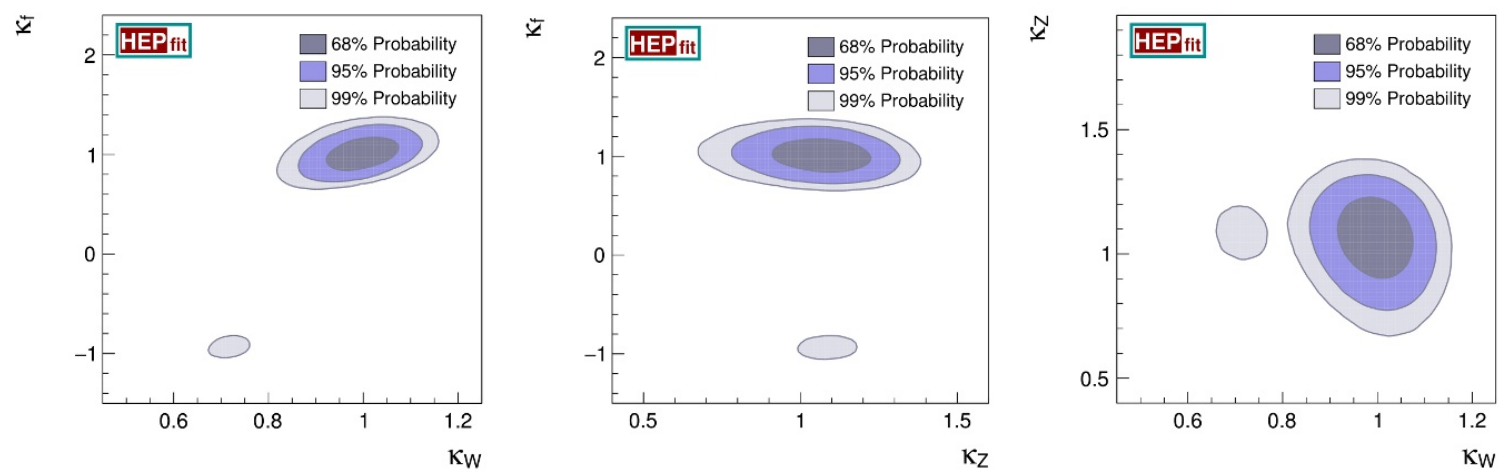

Figure 9. Two-dimensional probability distributions for $\kappa_{W}$ and $\kappa_{f}$ (left), for $\kappa_{Z}$ and $\kappa_{f}$ (center), and for $\kappa_{W}$ and $\kappa_{Z}$ (right) at $68 \%, 95 \%$, and $99 \%$ (darker to lighter), obtained from the fit to the Higgs-boson signal strengths. Note that a small region with $\kappa_{f}<0$ is still allowed at $99 \%$ probability.

We also consider the case in which we only lift fermion universality and introduce different rescaling factors for charged leptons $\left(\kappa_{\ell}\right)$, up-type quarks $\left(\kappa_{u}\right)$, and down-type quarks $\left(\kappa_{d}\right)$, while keeping a unique parameter $\kappa_{V}$ for both $H V V$ couplings. In this case, from the Higgs-boson signal strengths we obtain the constraints on the scale factors presented in table 14 and in the top plots of figure 10. By adding the EWPO to the fit, the constraints become stronger, as shown in table 15 and in the bottom plots of figure 10 . In this case, the Higgs-boson signal strengths are approximately symmetric under the exchanges $\kappa_{\ell} \leftrightarrow-\kappa_{\ell}, \kappa_{d} \leftrightarrow-\kappa_{d}$ and/or $\left\{\kappa_{V}, \kappa_{u}\right\} \leftrightarrow\left\{-\kappa_{V},-\kappa_{u}\right\}$. These approximate symmetries follow from the small effect of the interference between tau and/or bottomquark loops with top-quark/ $W$ loops in the Higgs-boson decay into two photons, as well as the relatively small interference between bottom- and top-quark loops in gluon-fusion, for $\left|\kappa_{V, u, d, \ell}\right| \sim 1$. Moreover, we find that negative values of $\kappa_{u}$ are disfavoured in the fit. Hence, in figure 10 we consider only the parameter space where all $\kappa$ 's are positive. Again, the results on table 13 correspond to the SM-like solution, i.e. $\kappa_{V, u, d, \ell}>0$.

Finally, we consider the case in which both the assumptions of custodial symmetry and fermion universality are lifted, and perform a five-parameter fit of $\kappa_{W}, \kappa_{Z}, \kappa_{\ell}, \kappa_{u}$, and $\kappa_{d}$ reported in table 16. Following the previous discussion, we restrict all the parameters but $\kappa_{u}$ (which has an important interference with $\kappa_{W}$ in $H \rightarrow \gamma \gamma$ ) to be positive.

The results presented in this section agree with the recent LHC combination of Higgs couplings in ref. [68], taking into account that the coupling to down quarks in our analysis also includes the Tevatron measurements. See also refs. [69-76] for other recent Higgs couplings analyses. 


\begin{tabular}{|c|c|c|c|}
\hline & Result & 95\% Prob. & Correlation Matrix \\
\hline$\kappa_{V}$ & $0.97 \pm 0.08$ & {$[0.80,1.13]$} & 1.00 \\
\hline$\kappa_{\ell}$ & $1.01 \pm 0.14$ & {$[0.73,1.30]$} & 0.541 .00 \\
\hline$\kappa_{u}$ & $0.97 \pm 0.13$ & {$[0.73,1.25]$} & $\begin{array}{lll}0.42 & 0.41 & 1.00\end{array}$ \\
\hline$\kappa_{d}$ & $0.91 \pm 0.21$ & {$[0.48,1.35]$} & $\begin{array}{llll}0.81 & 0.61 & 0.77 & 1.00\end{array}$ \\
\hline
\end{tabular}

Table 14. SM-like solution in the fit of $\kappa_{V}, \kappa_{\ell}, \kappa_{u}$, and $\kappa_{d}$ to the Higgs-boson signal strengths.
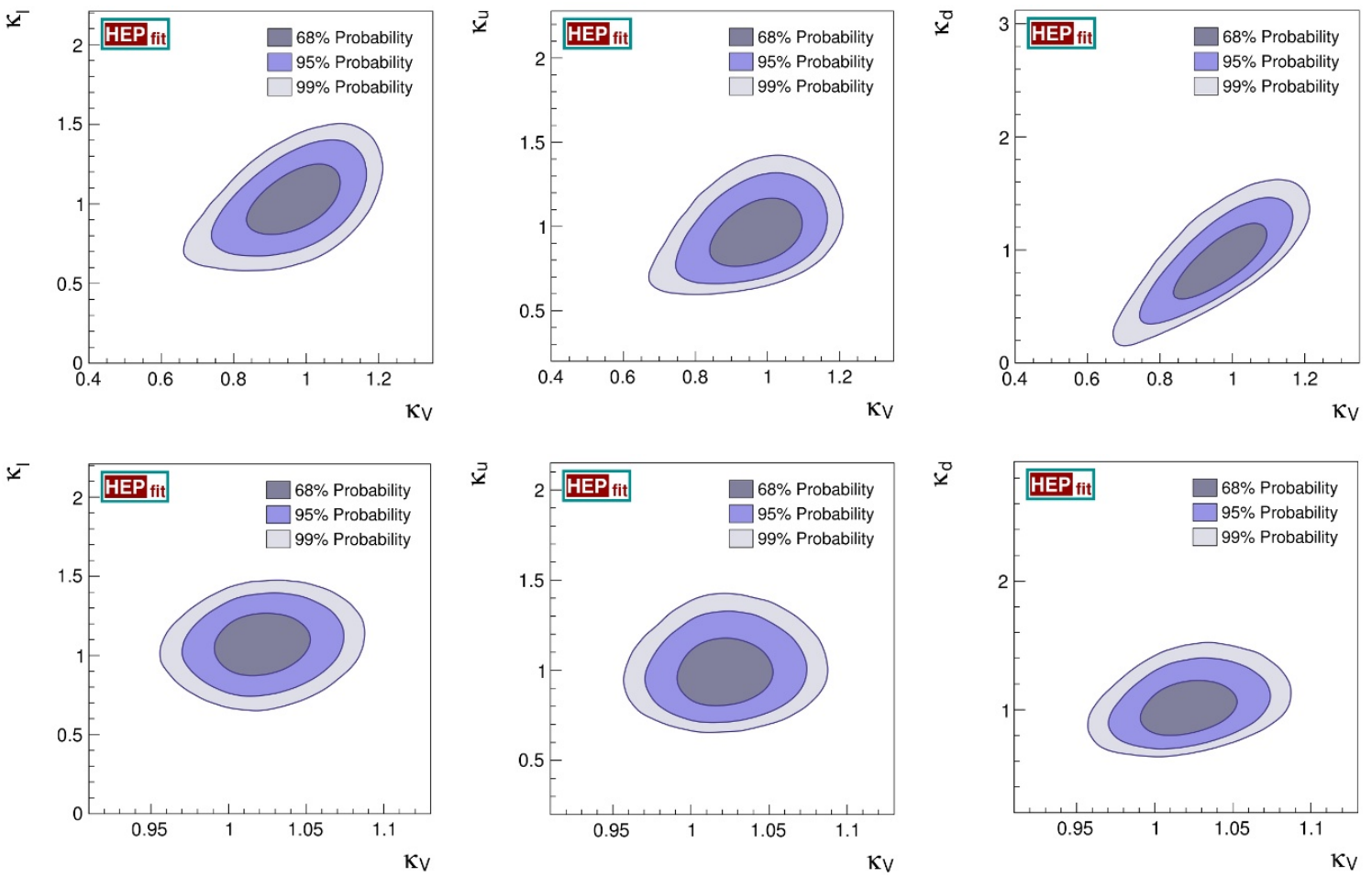

Figure 10. Two-dimensional probability distributions for $\kappa_{V}$ and $\kappa_{\ell}$, for $\kappa_{V}$ and $\kappa_{u}$, and for $\kappa_{V}$ and $\kappa_{d}$, at $68 \%, 95 \%$, and $99 \%$ (darker to lighter), obtained from the fit to the Higgs-boson signal strengths only (top plots) or the combination of Higgs-boson signal strengths and EWPO (bottom plots).

\begin{tabular}{|c|c|c|c|}
\hline & Result & 95\% Prob. & Correlation Matrix \\
\hline$\kappa_{V}$ & $1.02 \pm 0.02$ & {$[0.98,1.06]$} & 1.00 \\
\hline$\kappa_{\ell}$ & $1.07 \pm 0.12$ & {$[0.82,1.32]$} & $0.15 \quad 1.00$ \\
\hline$\kappa_{u}$ & $1.01 \pm 0.12$ & {$[0.79,1.27]$} & $\begin{array}{lll}0.10 & 0.24 & 1.00\end{array}$ \\
\hline$\kappa_{d}$ & $1.01 \pm 0.13$ & {$[0.76,1.30]$} & $\begin{array}{llll}0.31 & 0.38 & 0.78 & 1.00\end{array}$ \\
\hline
\end{tabular}

Table 15. Same as table 14, but considering both the Higgs-boson signal strengths and the EWPO. 


\begin{tabular}{|c|c|c|c|}
\hline & Result & 95\% Prob. & Correlation Matrix \\
\hline$\kappa_{W}$ & $0.94 \pm 0.10$ & {$[0.73,1.13]$} & 1.00 \\
\hline$\kappa_{Z}$ & $1.03 \pm 0.13$ & {$[0.77,1.28]$} & 0.341 .00 \\
\hline$\kappa_{\ell}$ & $1.02 \pm 0.15$ & {$[0.73,1.33]$} & $\begin{array}{lll}0.55 & 0.22 & 1.00\end{array}$ \\
\hline$\kappa_{u}$ & $0.95 \pm 0.13$ & {$[-0.96,-0.72] \cup[0.68,1.28]$} & $\begin{array}{llll}0.49 & 0.04 & 0.44 & 1.00\end{array}$ \\
\hline$\kappa_{d}$ & $0.91 \pm 0.22$ & {$[0.46,1.36]$} & $\begin{array}{lllll}0.81 & 0.36 & 0.62 & 0.78 & 1.00\end{array}$ \\
\hline
\end{tabular}

Table 16. Results of the simultaneous fit of $\kappa_{W}, \kappa_{Z}, \kappa_{\ell}, \kappa_{u}$, and $\kappa_{d}$, considering only Higgs-boson signal strengths.

\section{$6 \quad$ Expected sensitivities at future lepton colliders}

Future lepton colliders represent an opportunity to reach the ultimate precision both on EWPO and Higgs-boson couplings. In this work, we assess the impact of this improvement in precision by considering the following proposed $e^{+} e^{-}$colliders: the Future Circular Collider (FCCee) project at CERN [77], the International Linear Collider (ILC) in Japan [78, 79], and the Circular Electron Positron Collider (CepC) in China [80, 81]. For completeness in the comparison we also consider the improvements in the measurements of EWPO and Higgs-boson signal strengths expected at the High Luminosity LHC (HLLHC) [82-85]. In this section we describe the different physics scenarios we will consider, and estimate the improvements they offer in terms of sensitivity to the different NP models described in sections 4 and 5, comparing the results with those obtained using current data. See refs. [9, 86-89] for earlier analyses of this kind.

Across its years of operation, the FCCee design includes running at the $Z$ pole, and at the $W W, H Z$, and $t \bar{t}$ production thresholds, with the possibility of a dedicated run at center-of-mass energy $\sqrt{s} \gtrsim 350 \mathrm{GeV}$ to explore the top-quark couplings. Compared to other options for future $e^{+} e^{-}$colliders, the FCCee also offers the largest integrated luminosity and allows to assess an optimistic best-case scenario. The expected performance of the FCCee machine is documented in refs. [77, 90], and summarized in table 17. The values of integrated luminosity presented there are a useful baseline for our study. Further improvements in performance are under consideration, including an increase in center-ofmass energy. Within the context of our analyses, these improvements would further reduce the statistical uncertainties. On the other hand, since the precision on the observables considered in our study will be mainly dominated by the systematic uncertainties, our conclusions would still hold to a large extent.

The ILC project consists of a linear $e^{+} e^{-}$collider optimized for Higgs-boson and topquark precision measurements, and would initially run at energies $\sqrt{s}=250,350$, and $500 \mathrm{GeV}$ [78]. The current proposed scenarios would involve approximately 20 years of operation, including a luminosity upgrade. There is also the possibility of extending the energy reach of the machine up to $1 \mathrm{TeV}$, and we include this in our list of physics scenarios. The energy and luminosity settings of the Higgs-boson runs that we study in this work are given in table 18 [91]. Improved measurements of the properties of the $Z$ lineshape 


\begin{tabular}{|c|c|c|c|c|c|}
\hline FCCee & $Z$ pole & $\begin{array}{c}W W \\
\text { threshold }\end{array}$ & $\begin{array}{c}H Z \\
\text { threshold }\end{array}$ & $\begin{array}{c}t \bar{t} \\
\text { threshold }\end{array}$ & $\begin{array}{c}\text { Above } t \bar{t} \\
\text { threshold }\end{array}$ \\
\hline$\sqrt{s}[\mathrm{GeV}]$ & 90 & 160 & 240 & 350 & $>350$ \\
$\mathcal{L}\left[\mathrm{ab}^{-1} /\right.$ year $]$ & 88 & 15 & 3.5 & 1.0 & 1.0 \\
Years of operation & $0.3 / 2.5$ & 1 & 3 & 0.5 & 3 \\
\hline Events & $10^{12} / 10^{13}$ & $10^{8}$ & $2 \times 10^{6}$ & $2.1 \times 10^{5}$ & $7.5 \times 10^{4}$ \\
\hline
\end{tabular}

Table 17. Expected performances of the FCCee machine, taken from ref. [90].

\begin{tabular}{|c|ccc|ccc|}
\hline ILC & \multicolumn{3}{|c|}{ Phase 1 } & \multicolumn{3}{c|}{ Phase 2 } \\
& & & & \multicolumn{3}{c|}{ (Luminosity upgrade) } \\
\hline$\sqrt{s}[\mathrm{GeV}]$ & 250 & 500 & 1000 & 250 & 500 & 100 \\
$\int \mathcal{L} d t\left[\mathrm{ab}^{-1}\right]$ & 0.25 & 0.5 & 1 & 1.15 & 1.6 & 2.5 \\
$\int d t\left(10^{7} \mathrm{~s}\right)$ & 3 & 3 & 3 & 3 & 3 & 3 \\
\hline
\end{tabular}

Table 18. Expected performances of the ILC machine, taken from ref. [91].

at $\sqrt{s} \approx 91 \mathrm{GeV}$, on the other hand, would require a machine upgrade from the Technical Design Report to achieve an optimal luminosity performance [78]. We therefore do not consider this scenario here. As far as EWPO are concerned, we only include the improvements in the Higgs-boson, top-quark, and $W$ masses, where the latter is obtained from the measurements of $e^{+} e^{-} \rightarrow W^{+} W^{-}$above threshold with a target overall uncertainty at the level of approximately $3 \mathrm{MeV}$.

Finally, the CepC project is designed as a Higgs-boson and/or $Z$ factory [80, 81]. Running at $\sqrt{s} \approx 240 \mathrm{GeV}$ the CepC would produce about $10^{6}$ Higgs-boson particles, allowing measurements of its couplings at the percent level or better. During the $\sqrt{s} \approx$ $91 \mathrm{GeV}$ run, on the other hand, up to $10^{11} Z$ bosons could be produced, improving the sensitivity to the $Z$ couplings to the $10^{-4}$ level. With this statistics, the overall uncertainty for most observables is expected to be dominated by systematic effects. For the run at the $Z$-pole energy, we will assume a total integrated luminosity larger than $150 \mathrm{fb}^{-1}$, necessary to achieve the expected precision for all the different EWPO in table 4.1 of ref. [80, 81]. As in the case of the ILC, an improved measurement of the $W$ mass is possible at centerof-mass energies above the $W^{+} W^{-}$production threshold. For the $\sqrt{s}=250 \mathrm{GeV}$ run a direct $M_{W}$ measurement is expected with a similar uncertainty of approximately $3 \mathrm{MeV}$.

The expected experimental uncertainties on the different EWPO at the future colliders introduced above are summarized in table 19 [77, 79-81, 92]. When no input is provided for FCCee [77, 92], we adopted conservative numbers, depending on the experimental observable. The corresponding information for the expected accuracies in Higgs-boson signal strengths are summarized in table 20. In both tables 19 and 20, we have also included projections for the HL-LHC [82-85]. 
On the theory side, while the theoretical uncertainties associated to unknown higherorder corrections to EWPO in perturbation theory are subdominant compared with current experimental errors, this is no longer the case when we take into account the projected future experimental precision summarized in table 19. The present theoretical uncertainties for the most relevant EWPO are shown in table 21, where we compare them to the corresponding current and future experimental errors. It is clear that we need to improve SM calculations in order for theoretical uncertainties in the predictions of EWPO not to become a limiting factor at future experiments. The future projected theoretical errors in table 21 assume that the complete $\mathcal{O}\left(\alpha \alpha_{s}^{2}\right)$ corrections, the fermionic $\mathcal{O}\left(\alpha^{2} \alpha_{s}^{2}\right)$ and $\mathcal{O}\left(\alpha^{3}\right)$ corrections, and the leading 4-loop corrections entering via the $\rho$ parameter in the different observables will become available [87, 93, 94]. There are other sources of theoretical uncertainties not considered in the previous discussion. First, as explained in section 3, the parametric uncertainties on the theoretical predictions for the different EWPO receive important contributions from the current errors in the experimental measurements of $\Delta \alpha_{\text {had }}^{(5)}\left(M_{Z}\right)$ and $\alpha_{s}\left(M_{Z}\right)$ (see table 2). Apart from the experimental improvements summarized in table 19, we also assume in all future scenarios that a measurement of $\Delta \alpha_{\text {had }}^{(5)}$ is possible with a precision of $\pm 5 \times 10^{-5}$. Such an improvement is expected to be within the reach of ongoing and future experiments measuring the $e^{+} e^{-} \rightarrow$ hadrons cross section. This requires measuring the ratio $R$ of the hadronic to the muonic $e^{+} e^{-}$ cross sections with a relative uncertainty of $1 \%$ [95]. Likewise, for the strong coupling constant at the $Z$ pole, we use future lattice QCD projections, which estimate an uncertainty $\delta \alpha_{s}\left(M_{Z}\right)= \pm 0.0002[96]{ }^{4}$ Another observable which suffers of additional theoretical uncertainties is the top-quark mass. At $e^{+} e^{-}$colliders the top-quark mass can be extracted by reconstructing the $t \bar{t}$ production cross section in a scan around the production threshold. From the shape of the differential cross section one can derive the top-quark mass in different theoretically well-defined schemes, e.g. the potential-subtracted (PS) top-quark mass [98], or the so-called $1 S$ top-quark mass [99]. In both schemes the top-quark mass can be extracted with a theoretical uncertainty $\lesssim 50 \mathrm{MeV}[100,101]$, to be added to the projected experimental uncertainties shown in table 19. The relation between the PS or $1 S$ top-quark mass and the $\overline{\mathrm{MS}}$ top-quark mass has been calculated to 4 loops in perturbative QCD [102], and introduces an additional uncertainty of approximately $\sim 20 \mathrm{MeV}$ $(\sim 10 \mathrm{MeV})$ in the translation from the PS $(1 S)$ mass. In our fits we will assume a combined uncertainty in the top-quark mass of $50 \mathrm{MeV}$ for both the ILC and FCCee-t $\bar{t}$ scenarios.

In what follows we estimate the sensitivity to the different new physics scenarios at the above-mentioned future experiments. To do so, we assume that the future experimental measurements will be fully compatible with the SM predictions. In particular, we use the following reference values of the SM input parameters (see column Posterior in table 1),

$$
\begin{aligned}
m_{H} & =125.09 \mathrm{GeV}, \quad m_{t}=173.61 \mathrm{GeV}, \quad M_{Z}=91.1879 \mathrm{GeV}, \\
\alpha_{s}\left(M_{Z}\right) & =0.1180 \text { and } \Delta \alpha_{\text {had }}^{(5)}\left(M_{Z}\right)=0.02747,
\end{aligned}
$$

and take as errors the ones given in tables 19 and 20. In our analysis we assume that the theoretical calculations necessary to match the experimental precision will be available, and

\footnotetext{
${ }^{4}$ Based on [97].
} 


\begin{tabular}{|c|c|c|c|c|c|c|}
\hline & $\begin{array}{c}\text { Current } \\
\text { Data }\end{array}$ & HL-LHC & ILC & & $\begin{array}{l}\text { Cee } \\
\qquad \text { (Run) }\end{array}$ & CepC \\
\hline$\alpha_{s}\left(M_{Z}\right)$ & $0.1179 \pm 0.0012$ & & & & & \\
\hline$\Delta \alpha_{\text {had }}^{(5)}\left(M_{Z}\right)$ & $0.02750 \pm 0.00033$ & & & & & \\
\hline$M_{Z}[\mathrm{GeV}]$ & $91.1875 \pm 0.0021$ & & & \pm 0.0001 & $(\mathrm{FCCee}-Z)$ & \pm 0.0005 \\
\hline$m_{t}[\mathrm{GeV}]$ & $173.34 \pm 0.76$ & \pm 0.6 & \pm 0.017 & \pm 0.014 & $(\mathrm{FCCee}-t \bar{t})$ & \\
\hline$m_{H}[\mathrm{GeV}]$ & $125.09 \pm 0.24$ & \pm 0.05 & \pm 0.015 & \pm 0.007 & $(\mathrm{FCCee}-H Z)$ & \pm 0.0059 \\
\hline$M_{W}[\mathrm{GeV}]$ & $80.385 \pm 0.015$ & \pm 0.011 & \pm 0.0024 & \pm 0.001 & $($ FCCee- $W W)$ & \pm 0.003 \\
\hline$\Gamma_{W}[\mathrm{GeV}]$ & $2.085 \pm 0.042$ & & & \pm 0.005 & $($ FCCee- $W W)$ & \\
\hline$\Gamma_{Z}[\mathrm{GeV}]$ & $2.4952 \pm 0.0023$ & & & \pm 0.0001 & $($ FCCee- $Z)$ & \pm 0.0005 \\
\hline$\sigma_{h}^{0}[\mathrm{nb}]$ & $41.540 \pm 0.037$ & & & \pm 0.025 & $(\mathrm{FCCee}-Z)$ & \\
\hline $\sin ^{2} \theta_{\mathrm{eff}}^{\mathrm{lept}}$ & $0.2324 \pm 0.0012$ & & & \pm 0.0001 & $(\mathrm{FCCee}-Z)$ & \pm 0.000023 \\
\hline$P_{\tau}^{\text {pol }}$ & $0.1465 \pm 0.0033$ & & & \pm 0.0002 & $($ FCCee- $Z$ ) & \\
\hline $\mathcal{A}_{\ell}$ & $0.1513 \pm 0.0021$ & & & \pm 0.000021 & $($ FCCee- $Z[\mathrm{pol}])$ & \\
\hline $\mathcal{A}_{c}$ & $0.670 \pm 0.027$ & & & \pm 0.01 & $(\mathrm{FCCee}-Z[\mathrm{pol}])$ & \\
\hline $\mathcal{A}_{b}$ & $0.923 \pm 0.020$ & & & \pm 0.007 & $(\mathrm{FCCee}-Z[\mathrm{pol}])$ & \\
\hline$A_{\mathrm{FB}}^{0, \ell}$ & $0.0171 \pm 0.0010$ & & & \pm 0.0001 & $($ FCCee- $Z$ ) & \pm 0.0010 \\
\hline$A_{\mathrm{FB}}^{0, c}$ & $0.0707 \pm 0.0035$ & & & \pm 0.0003 & $(\mathrm{FCCee}-Z)$ & \\
\hline$A_{\mathrm{FB}}^{0, b}$ & $0.0992 \pm 0.0016$ & & & \pm 0.0001 & $($ FCCee- $Z)$ & \pm 0.00014 \\
\hline$R_{\ell}^{0}$ & $20.767 \pm 0.025$ & & & \pm 0.001 & $(\mathrm{FCCee}-Z)$ & \pm 0.007 \\
\hline$R_{c}^{0}$ & $0.1721 \pm 0.0030$ & & & \pm 0.0003 & $(\mathrm{FCCee}-Z)$ & \\
\hline$R_{b}^{0}$ & $0.21629 \pm 0.00066$ & & & \pm 0.00006 & $(\mathrm{FCCee}-Z)$ & \pm 0.00018 \\
\hline
\end{tabular}

Table 19. Expected experimental sensitivities to the different EWPO at future colliders. Apart from the improvements quoted in this table, we also assume that future measurements of $\Delta \alpha_{\text {had }}^{(5)}\left(M_{Z}\right)$ and $\alpha_{S}\left(M_{Z}\right)$, whose errors dominate in the parametric uncertainties of the theoretical predictions, are possible with an error of approximately $\pm 5 \times 10^{-5}$ and \pm 0.0002 , respectively. This assumption is particularly relevant for the FCCee and CepC fits, where the experimental precision for the bulk of electroweak precision measurements will be largely improved.

\begin{tabular}{|c|c|c|c|c|c|c|c|c|c|c|}
\hline & \multirow[t]{3}{*}{ Current } & \multirow[t]{3}{*}{ HL-LHC } & \multicolumn{6}{|c|}{ ILC } & \multirow[t]{3}{*}{ FCCee } & \multirow[t]{3}{*}{ CepC } \\
\hline & & & \multicolumn{3}{|c|}{ Phase 1} & \multicolumn{3}{|c|}{ Phase 2} & & \\
\hline & & & 250 & 500 & 1000 & 250 & 500 & 1000 & & \\
\hline$H \rightarrow b \bar{b}$ & $\gtrsim 23 \%$ & $5-36 \%$ & $1.2 \%$ & $1.8-28 \%$ & $0.3-6 \%$ & $0.56 \%$ & $0.37-16 \%$ & $0.3-3.8 \%$ & $0.2-0.6 \%$ & $0.28 \%$ \\
\hline$H \rightarrow c \bar{c}$ & & & $8.3 \%$ & $6.2-13 \%$ & $3.1 \%$ & $3.9 \%$ & $3.5-7.2 \%$ & $2 \%$ & $1.2 \%$ & $2.2 \%$ \\
\hline$H \rightarrow g g$ & & & $7 \%$ & $4.1-11 \%$ & $2.3 \%$ & $3.3 \%$ & $2.3-6 \%$ & $1.4 \%$ & $1.4 \%$ & $1.6 \%$ \\
\hline$H \rightarrow W W$ & $\gtrsim 15 \%$ & $4-11 \%$ & $6.4 \%$ & $2.4-9.2 \%$ & $1.6 \%$ & $3 \%$ & $1.3-5.1 \%$ & $1 \%$ & $0.9 \%$ & $1.5 \%$ \\
\hline$H \rightarrow \tau \tau$ & $\gtrsim 25 \%$ & $5-15 \%$ & $4.2 \%$ & $5.4-9 \%$ & $3.1 \%$ & $2 \%$ & $3-5 \%$ & $2 \%$ & $0.7 \%$ & $1.2 \%$ \\
\hline$H \rightarrow Z Z$ & $\gtrsim 24 \%$ & $4-17 \%$ & $19 \%$ & $8.2-25 \%$ & $4.1 \%$ & $8.8 \%$ & $4.6-14 \%$ & $2.6 \%$ & $3.1 \%$ & $4.3 \%$ \\
\hline$H \rightarrow \gamma \gamma$ & $\gtrsim 20 \%$ & $4-28 \%$ & $38 \%$ & $20-38 \%$ & $7 \%$ & $16 \%$ & $13-19 \%$ & $5.4 \%$ & $3.0 \%$ & $9 \%$ \\
\hline$H \rightarrow Z_{\gamma}$ & & $10-27 \%$ & & & & & & & & \\
\hline$H \rightarrow \mu \mu$ & & $14-23 \%$ & & & $31 \%$ & & & $20 \%$ & $13 \%$ & $17 \%$ \\
\hline
\end{tabular}

Table 20. Future expected sensitivity to Higgs-boson observables at various future colliders considered in this study. 


\begin{tabular}{|lcccccc|}
\hline Observable & $\begin{array}{c}\text { Current } \\
\text { Th. Error }\end{array}$ & $\begin{array}{c}\text { Future } \\
\text { Th. Error }\end{array}$ & $\begin{array}{c}\text { Current } \\
\text { Exp. Error }\end{array}$ & ILC & FCC-ee & CepC \\
\hline$M_{W}[\mathrm{MeV}]$ & 4 & 1 & 15 & $3-4$ & 1 & 3 \\
$\sin ^{2} \theta_{\text {eff }}^{\text {lept }}\left[10^{-5}\right]$ & 4.5 & 1.5 & 16 & & 0.6 & 2.3 \\
$\Gamma_{Z}[\mathrm{MeV}]$ & 0.5 & 0.2 & 2.3 & & 0.1 & 0.5 \\
$R_{b}^{0}\left[10^{-5}\right]$ & 15 & 10 & 66 & & 6 & 17 \\
\hline
\end{tabular}

Table 21. Projected theoretical uncertainty for the different EWPO and comparison with the corresponding experimental sensitivity at various future colliders considered in this study.

in our fits we use the future projected uncertainties in table 21. To illustrate the impact of theoretical uncertainties, we also consider another scenario where, as in the current EWPO fit, theoretical uncertainties are subdominant and are neglected in the analysis. In this scenario we also assume that the only uncertainty affecting the top-quark mass parameter is the one given in table 19 .

With these settings we have performed fits to the main NP scenarios studied in sections 4 and 5, and compared the results with those obtained in a fit assuming the errors of current data. ${ }^{5}$ The results of the fits to EWPO only are summarized in table 22, while those from the fits to EWPO plus Higgs-boson observables are reported in table 23. In these tables we illustrate the sensitivity to each NP parameter introduced in sections 4 and 5 by showing the 1- $\sigma$ uncertainty on the corresponding parameter from the fit. A comparison of the projected sensitivity on EW parameters and Higgs coupling constants for various future colliders is shown in figure 11 .

From the results in table 22 we observe how the FCCee, with dedicated runs aimed at improving the measurements of the different EWPO, offers the best performance in terms of constraints on NP. We show the results obtained with the $Z$-pole runs, with and without polarization, and also show the effect of adding the improved measurement of the $W$ mass ( $W W$ column) as well as the sensitivity reached after the completion of the whole FCCee program (tt column). Several things are apparent from this table. The first one is that, for the NP models considered here, the use of polarized beams at the FCCee would have only a minor impact on the constraining power of the machine. Looking into the results for the different models we observe how, as expected, the major improvement in sensitivity comes from the more precise properties of the $Z$ lineshape. After this first run, one can still achieve notable improvements in the sensitivity to the $U$ parameter $\left(\delta \varepsilon_{2}\right)$ from the measurement of $M_{W}$ (notice that this is essentially the only EWPO that depends on $U$ ). Likewise, the sensitivity to $\kappa_{V}$ can be reduced by a factor of $\sim 2$ with the measurement of $m_{t}$. This can be understood from eq. (5.3), the lower-right panel of figure 3, and the positive correlation between $M_{W}$ and $m_{t}$.

In general, the FCCee program would improve current constraints by about an order of magnitude. The CepC also offers good prospects to obtain more stringent NP constraints

\footnotetext{
${ }^{5}$ For consistency in the comparison, in this fit we also set the central values to the SM predictions summarized in eq. (6.1).
} 
from EWPO. However, given the information currently available about the machine performance, the CepC bounds would only be a factor of approximately $4-5$ better than the bounds derived from current EWPO. Notice also that the current physics program lacks a dedicated run to improve the measurement of the top-quark mass, which plays a significant role in some cases as explained above. In fact, at the ILC, even without a dedicated run at the $Z$ pole, the precise determinations of $M_{W}$ and $m_{t}$ are enough to reach the same sensitivity to $\kappa_{V}$ as at the CepC.

In table 22 we have also illustrated the effect of the theoretical uncertainties in the results of electroweak fits with the information from future $e^{+} e^{-}$colliders. In this table, the results in the columns with grey background have been computed using the projected theoretical uncertainties, while such uncertainties have been neglected in the columns with white background. As one expects from looking at table 21, the effect of the future theoretical uncertainties on the $\mathrm{CepC}$ results are mild, but they are clearly non-negligible compared to the FCCee precision. Indeed, in the case of the FCCee, theoretical uncertainties can reduce the sensitivity to NP in some cases by up to a factor of 2 compared to cases in which the theoretical errors are subdominant.

Finally, in table 23, we show the level of sensitivity to modified Higgs-boson couplings achievable at the various future colliders considered in this study, in the different scenarios explained in section 5. In this case, let us emphasize however that, even before any future lepton collider, the HL-LHC will provide much better determinations of the Higgs-boson properties compared to what has been so far obtained with current data. Using the fermionuniversal custodial-symmetric scenario as a reference, i.e. $\kappa_{W}=\kappa_{Z} \equiv \kappa_{V}$ and $\kappa_{u}=\kappa_{d}=$ $\kappa_{\ell} \equiv \kappa_{f}$, the HL-LHC would be twice as sensitive to deviations from $\kappa_{V}=1$, and up to 6-7 times as sensitive to deviations from $\kappa_{f}=1$. These results would be further improved at lepton colliders by a factor of $9(5)$ for $\kappa_{V}\left(\kappa_{f}\right)$. The much larger gain in sensitivity for $\kappa_{f}$ than for $\kappa_{V}$ can be understood by noticing that the measurement of Higg-boson couplings to vector bosons will be systematic dominated within the current LHC program, while the measurement of Higgs-boson couplings to fermions will need the full HL-LHC luminosity for the systematic uncertainty to be comparable to the statistical one. Focusing on the results obtained at the different lepton colliders we observe how, assuming custodial symmetry, the FCCee would offer a somewhat better performance than CepC in terms of measuring the Higgs-boson couplings to both vector bosons and fermions (in part because of the more precise determination of $\kappa_{V}$ via EWPO). At the ILC the results indicate that, again assuming custodial symmetry, the initial phase would not be enough to match the FCCee or CepC precision. Matching the CepC would be possible after a luminosity upgrade even in the absence of a dedicated run at $\sqrt{s}=1 \mathrm{TeV}$. Including such a run in the physics program would make the ILC performances comparable to the FCCee physics reach for this scenario. For the scenario with $\kappa_{Z} \neq \kappa_{W}$ we observe that, while the CepC Higgs-boson run will only explore center-of-mass energies $\sqrt{s} \approx 240 \mathrm{GeV}$, where Higgs-boson production occurs mostly via $Z H$ associated production, running at the FCCee with $\sqrt{s}=350 \mathrm{GeV}$ or at the ILC with $\sqrt{s}=500 \mathrm{GeV}$ or $\sqrt{s}=1000 \mathrm{GeV}$ gives also access to $W$-boson fusion production (as well as $t \bar{t} H$ associated production in the ILC case). This results in a FCCee (ILC) determination of $\kappa_{W}$ approximately 3 (10) times more precise than at the CepC. 
害导恶富 刍 $\exists$ :

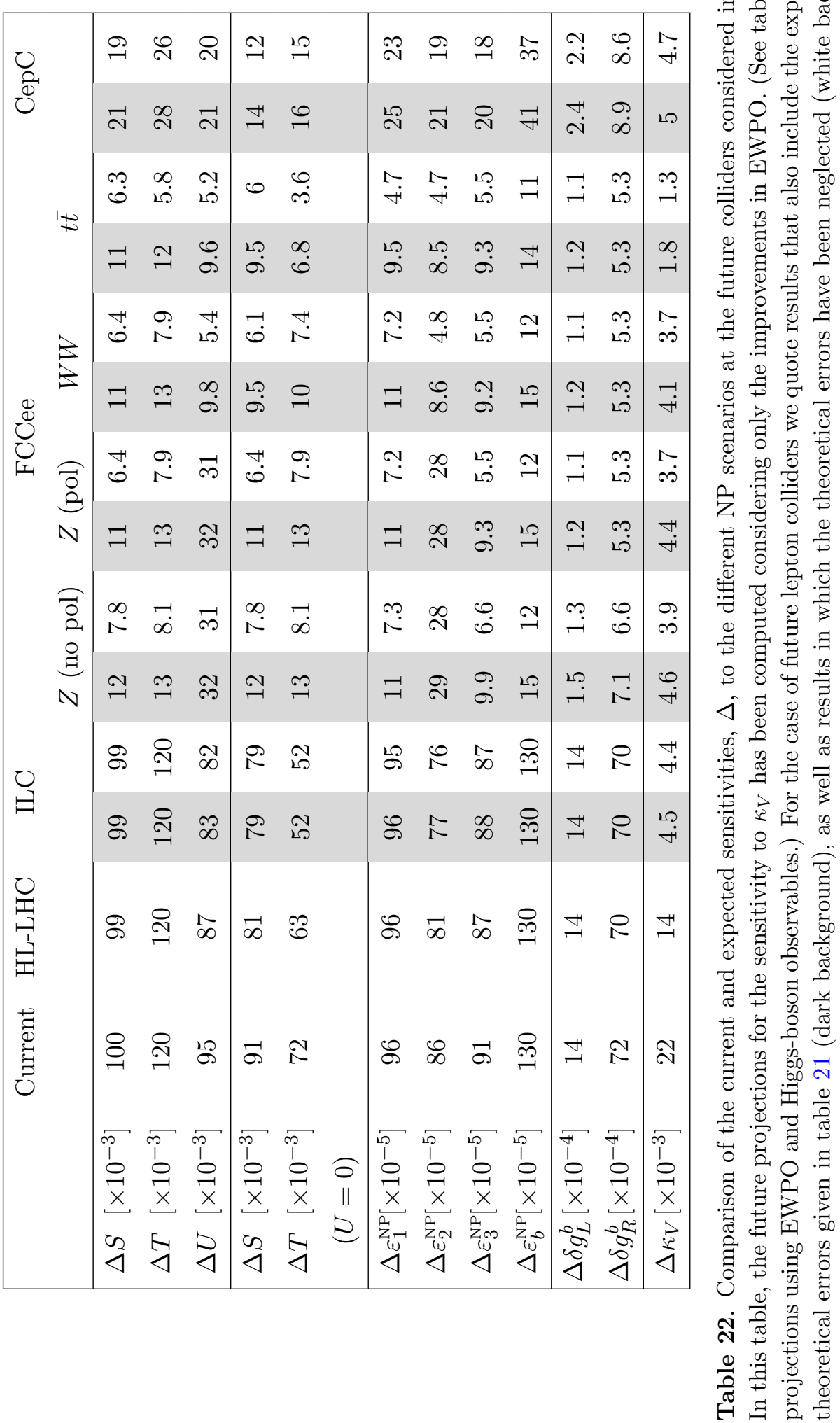




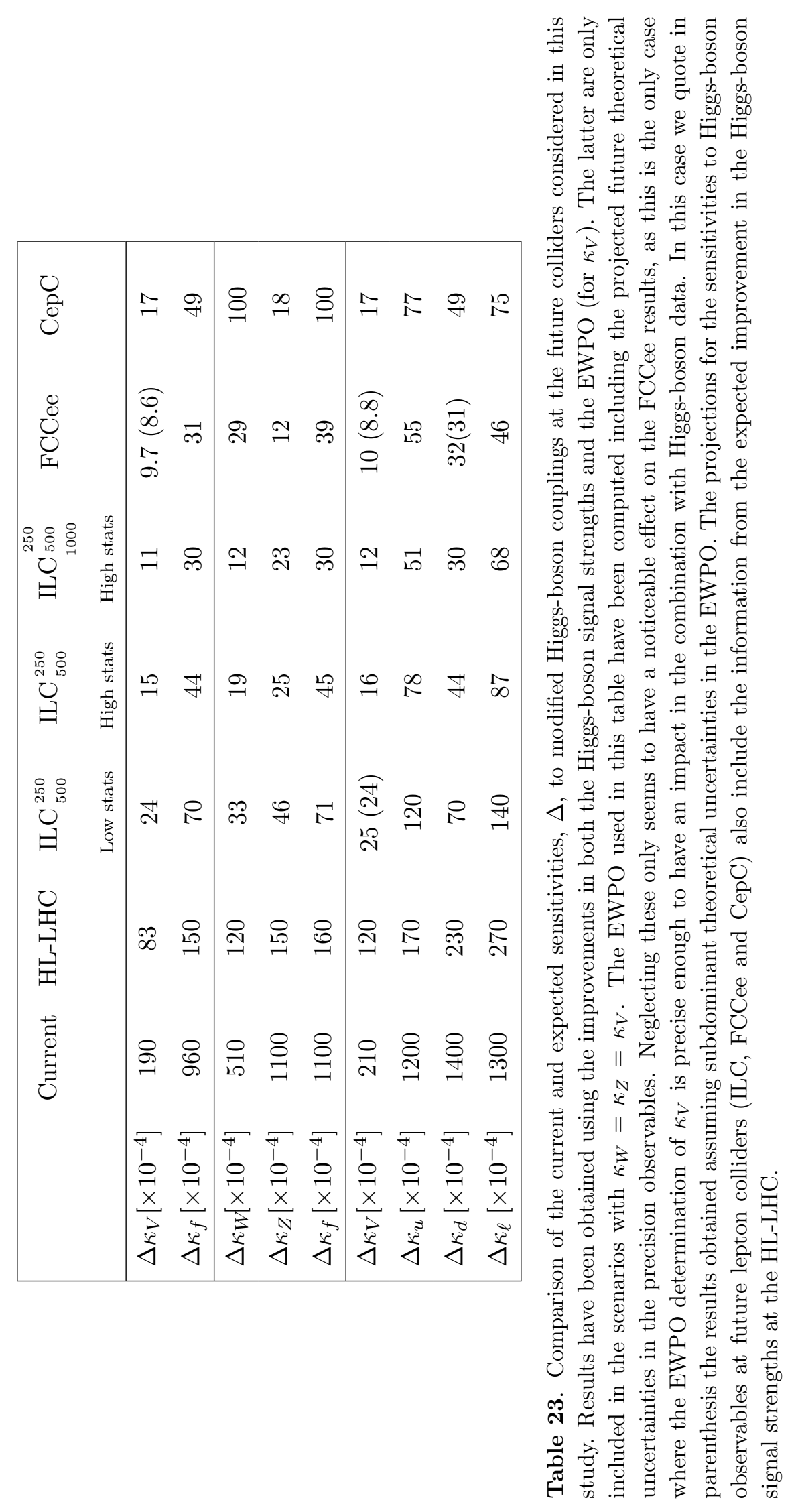



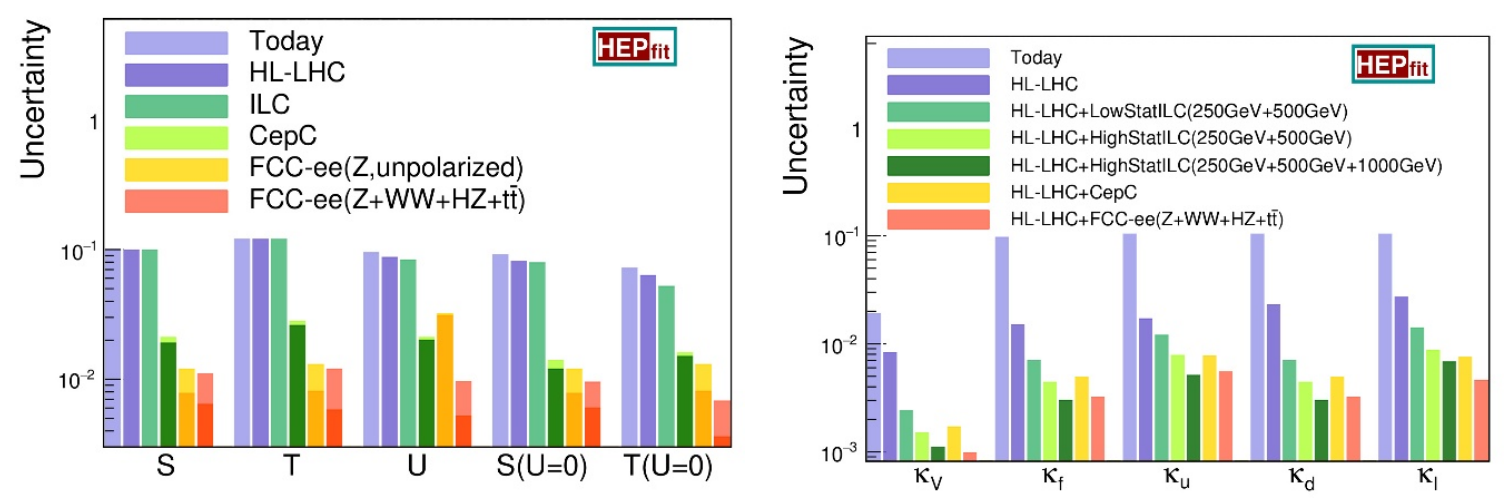

Figure 11. Comparison of expected sensitivities to oblique parameters (left) and modified Higgs couplings (right) from future collider experiments. Different shades of the same colour correspond to results including or neglecting the future theoretical uncertainties.

\section{Conclusions}

With the discovery of a SM-like Higgs boson during Run I of the LHC, the possibility of using EW and Higgs-boson precision measurements as a portal to NP has become a reality. Through the steady improvement of both theoretical and experimental accuracies, electroweak and Higgs-boson precision physics could lead us a long way towards determining the UV completion of the SM and the more fundamental origin of the spontaneously-broken realization of the electroweak symmetry.

Indirect searches for NP are indeed as important as ever in the physics program of Run II of the LHC: they will probe physics at inaccessible high scales and provide clues on the nature of new particles. In this context, it is very valuable, if not essential, to provide a complete and consistent framework in which all available experimental data, from precision measurements of electroweak observables and Higgs-boson couplings to flavourphysics results, can be analyzed to constrain the theory in a statistically significant way.

The study presented in this paper illustrates how this can be achieved in the context of the HEPfit package, and provides results for a global fit of EWPO and Higgs-boson signal-strength measurements obtained from LHC Run I data collected at 7 and $8 \mathrm{TeV}$.

At the moment, the constraints derived for Higgs-boson couplings to SM gauge bosons and fermions are overall compatible with SM predictions within the current accuracy. From the results of section 5 we see that the combined study of EWPO and Higgs-boson observables can provide more stringent constraints on Higgs-boson couplings. We can foresee that the higher statistics expected in Run II of the LHC will offer the possibility to isolate potential NP effects from global fits of SM precision observables. This will become even more crucial at the HL-LHC and at a future generation of $e^{+} e^{-}$colliders (FCCee, ILC, CepC) where very high experimental precision for EWPO and Higgs-boson couplings will be achievable. We have dedicated a section of this paper to a study of the sensitivity of different future experimental facilities to NP effects, and have determined at what point more accurate theoretical predictions will be needed (see also ref. [103]). 
Finally, we notice that deviations from the SM predictions of EWPO and Higgs-boson couplings constitute indirect evidence of new physics that still need to be interpreted in terms of specific physical degrees of freedom. A more refined theoretical approach, which entails a generalization of the SM Lagrangian to systematically include all effective interactions generated by the presence of NP at the UV scale, will then be necessary in order to explore the nature of such deviations. In a following paper [8] we will explore the possibility of using an effective field theory approach to build a model-independent study of NP effects in Higgs-boson couplings and use the HEPfit framework to combine it with a fit of all available electroweak precision data.

\section{Acknowledgments}

We thank P. Janot for pointing out an omission in the list of FCCee measurements taken as input. M. C. is associated to the Dipartimento di Matematica e Fisica, Università di Roma Tre, and E. F. and L. S. are associated to the Dipartimento di Fisica, Università di Roma "La Sapienza". The research leading to these results has received funding from the European Research Council under the European Union's Seventh Framework Programme (FP/2007-2013) / grants n. 267985 and n. 279972. The work of L. R. is supported in part by the U.S. Department of Energy under grant DE-SC0010102, and by the National Science Foundation under Grant No. NSF PHY11-25915. L. R. would like to thanks the Kavli Institute for Theoretical Physics (UCSB) for hospitality while this work was being completed.

Open Access. This article is distributed under the terms of the Creative Commons Attribution License (CC-BY 4.0), which permits any use, distribution and reproduction in any medium, provided the original author(s) and source are credited.

\section{References}

[1] HEPFIT collaboration, HEPfit: a code for the combination of indirect and direct constraints on high energy physics models, in preparation.

[2] HEPfit collaboration webpage, http://hepfit.roma1.infn.it.

[3] M. Ciuchini, E. Franco, S. Mishima and L. Silvestrini, Electroweak precision observables, new physics and the nature of a 126 GeV Higgs boson, JHEP 08 (2013) 106 [arXiv:1306.4644] [INSPIRE].

[4] J. de Blas et al., Global Bayesian analysis of the Higgs-boson couplings, in International Conference on High Energy Physics 2014 (ICHEP 2014), Valencia Spain July 2-9 2014 [Nucl. Part. Phys. Proc. 273-275 (2016) 834] [arXiv:1410.4204] [InSPIRE].

[5] M. Ciuchini, E. Franco, S. Mishima, M. Pierini, L. Reina and L. Silvestrini, Update of the electroweak precision fit, interplay with Higgs-boson signal strengths and model-independent constraints on new physics, in International Conference on High Energy Physics 2014 (ICHEP 2014), Valencia Spain July 2-9 2014 [arXiv:1410.6940] [INSPIRE].

[6] L. Reina et al., Precision constraints on non-standard Higgs-boson couplings with HEPfit, PoS (EPS-HEP2015) 187 [INSPIRE]. 
[7] J. de Blas et al., Updates on fits to electroweak parameters, in Proceedings of $27^{\text {th }}$ International Symposium on Lepton Photon Interactions at High Energy (LP15), Ljubljana Slovenia August 17-22 2015 [PoS (LeptonPhoton2015)013] [INSPIRE].

[8] J. de Blas et al., in preparation.

[9] GFitTer Group collaboration, M. Baak et al., The global electroweak fit at NNLO and prospects for the LHC and ILC, Eur. Phys. J. C 74 (2014) 3046 [arXiv:1407.3792] [INSPIRE].

[10] Particle Data Group collaboration, K.A. Olive et al., Review of particle physics, Chin. Phys. C 38 (2014) 090001 [INSPIRE].

[11] A. Caldwell, D. Kollar and K. Kroninger, BAT: the Bayesian Analysis Toolkit, Comput. Phys. Commun. 180 (2009) 2197 [arXiv:0808.2552] [INSPIRE].

[12] A. Akhundov, A. Arbuzov, S. Riemann and T. Riemann, The ZFITTER project, Phys. Part. Nucl. 45 (2014) 529 [arXiv:1302.1395] [INSPIRE].

[13] H. Burkhardt and B. Pietrzyk, Recent BES measurements and the hadronic contribution to the QED vacuum polarization, Phys. Rev. D 84 (2011) 037502 [arXiv:1106.2991] [INSPIRE].

[14] Sld Electroweak Group, Delphi, AlEPH, SLD, SLD Heavy Flavour Group, OPAL, LEP Electroweak Working Group and L3 collaborations, S. Schael et al., Precision electroweak measurements on the $Z$ resonance, Phys. Rept. 427 (2006) 257 [hep-ex/0509008] [INSPIRE].

[15] ATLAS, CDF, CMS and D0 collaborations, First combination of Tevatron and LHC measurements of the top-quark mass, arXiv:1403.4427 [INSPIRE].

[16] ATLAS and CMS collaborations, Combined measurement of the Higgs boson mass in pp collisions at $\sqrt{s}=7$ and 8 TeV with the ATLAS and CMS experiments, Phys. Rev. Lett. 114 (2015) 191803 [arXiv:1503.07589] [INSPIRE].

[17] Tevatron Electroweak Working Group, CDF and D0 collaborations, 2012 update of the combination of CDF and D0 results for the mass of the $W$ boson, arXiv:1204.0042 [INSPIRE].

[18] Tevatron Electroweak Working Group, CDF, DelPhi, SLD Electroweak and Heavy Flavour Groups, AlePH, LEP Electroweak Working Group, SLD, OPAL, D0 and L3 collaboration, Precision electroweak measurements and constraints on the Standard Model, arXiv:1012.2367 [INSPIRE].

[19] CDF collaboration, T.A. Aaltonen et al., Measurement of $\sin ^{2} \theta_{\mathrm{eff}}^{\text {lept }}$ using $e^{+} e^{-}$pairs from $\gamma^{*} / Z$ bosons produced in pp collisions at a center-of-momentum energy of $1.96 \mathrm{TeV}$, Phys. Rev. D 93 (2016) 112016 [arXiv:1605.02719] [INSPIRE].

[20] CDF collaboration, T.A. Aaltonen et al., Indirect measurement of $\sin ^{2} \theta_{W}$ (or $M_{W}$ ) using $\mu^{+} \mu^{-}$pairs from $\gamma^{*} / Z$ bosons produced in p $\bar{p}$ collisions at a center-of-momentum energy of 1.96 TeV, Phys. Rev. D 89 (2014) 072005 [arXiv: 1402.2239] [InSPIRE].

[21] D0 collaboration, V.M. Abazov et al., Measurement of the effective weak mixing angle in $p \bar{p} \rightarrow Z / \gamma^{*} \rightarrow e^{+} e^{-}$events, Phys. Rev. Lett. 115 (2015) 041801 [arXiv:1408.5016] [INSPIRE]. 
[22] ATLAS collaboration, Measurement of the forward-backward asymmetry of electron and muon pair-production in pp collisions at $\sqrt{s}=7 \mathrm{TeV}$ with the ATLAS detector, JHEP 09 (2015) 049 [arXiv: 1503.03709] [INSPIRE].

[23] CMS collaboration, Measurement of the weak mixing angle with the Drell-Yan process in proton-proton collisions at the LHC, Phys. Rev. D 84 (2011) 112002 [arXiv:1110.2682] [INSPIRE].

[24] LHCb collaboration, Measurement of the forward-backward asymmetry in $Z / \gamma^{*} \rightarrow \mu^{+} \mu^{-}$ decays and determination of the effective weak mixing angle, JHEP 11 (2015) 190 [arXiv: 1509.07645] [INSPIRE].

[25] A. Freitas, Higher-order electroweak corrections to the partial widths and branching ratios of the $Z$ boson, JHEP 04 (2014) 070 [arXiv:1401.2447] [INSPIRE].

[26] Y. Schröder and M. Steinhauser, Four-loop singlet contribution to the $\rho$ parameter, Phys. Lett. B 622 (2005) 124 [hep-ph/0504055] [INSPIRE].

[27] K.G. Chetyrkin, M. Faisst, J.H. Kuhn, P. Maierhofer and C. Sturm, Four-loop QCD corrections to the $\rho$ parameter, Phys. Rev. Lett. 97 (2006) 102003 [hep-ph/0605201] [INSPIRE].

[28] R. Boughezal and M. Czakon, Single scale tadpoles and $O\left(G_{F} m_{t}^{2} \alpha_{s}^{3}\right)$ corrections to the $\rho$ parameter, Nucl. Phys. B 755 (2006) 221 [hep-ph/0606232] [INSPIRE].

[29] M. Awramik, M. Czakon, A. Freitas and G. Weiglein, Precise prediction for the $W$ boson mass in the Standard Model, Phys. Rev. D 69 (2004) 053006 [hep-ph/0311148] [InSPIRE].

[30] CMS collaboration, Determination of the top-quark pole mass and strong coupling constant from the t $\bar{t}$ production cross section in pp collisions at $\sqrt{s}=7$ TeV, Phys. Lett. B 728 (2014) 496 [Erratum ibid. B 738 (2014) 526] [arXiv:1307.1907] [INSPIRE].

[31] S. Aoki et al., Review of lattice results concerning low-energy particle physics, arXiv: 1607.00299 [INSPIRE].

[32] M.E. Peskin and T. Takeuchi, A new constraint on a strongly interacting Higgs sector, Phys. Rev. Lett. 65 (1990) 964 [INSPIRE].

[33] M.E. Peskin and T. Takeuchi, Estimation of oblique electroweak corrections, Phys. Rev. D 46 (1992) 381 [INSPIRE].

[34] G. Altarelli and R. Barbieri, Vacuum polarization effects of new physics on electroweak processes, Phys. Lett. B 253 (1991) 161 [INSPIRE].

[35] G. Altarelli, R. Barbieri and S. Jadach, Toward a model independent analysis of electroweak data, Nucl. Phys. B 369 (1992) 3 [Erratum ibid. B 376 (1992) 444] [INSPIRE].

[36] G. Altarelli, R. Barbieri and F. Caravaglios, Nonstandard analysis of electroweak precision data, Nucl. Phys. B 405 (1993) 3 [inSPIRE].

[37] R. Barbieri, A. Pomarol, R. Rattazzi and A. Strumia, Electroweak symmetry breaking after LEP-1 and LEP-2, Nucl. Phys. B 703 (2004) 127 [hep-ph/0405040] [INSPIRE].

[38] D. Choudhury, T.M.P. Tait and C.E.M. Wagner, Beautiful mirrors and precision electroweak data, Phys. Rev. D 65 (2002) 053002 [hep-ph/0109097] [INSPIRE].

[39] C. Grojean, O. Matsedonskyi and G. Panico, Light top partners and precision physics, JHEP 10 (2013) 160 [arXiv:1306.4655] [INSPIRE]. 
[40] D. Ghosh, M. Salvarezza and F. Senia, Extending the analysis of electroweak precision constraints in composite Higgs models, Nucl. Phys. B 914 (2017) 346 [arXiv:1511.08235] [INSPIRE].

[41] ATLAS collaboration, Measurement of Higgs boson production in the diphoton decay channel in pp collisions at center-of-mass energies of 7 and 8 TeV with the ATLAS detector, Phys. Rev. D 90 (2014) 112015 [arXiv:1408.7084] [InSPIRE].

[42] CMS collaboration, Observation of the diphoton decay of the Higgs boson and measurement of its properties, Eur. Phys. J. C 74 (2014) 3076 [arXiv:1407.0558] [InSPIRE].

[43] ATLAS collaboration, Evidence for the Higgs-boson Yukawa coupling to $\tau$ leptons with the ATLAS detector, JHEP 04 (2015) 117 [arXiv: 1501.04943] [INSPIRE].

[44] CMS collaboration, Evidence for the $125 \mathrm{GeV}$ Higgs boson decaying to a pair of $\tau$ leptons, JHEP 05 (2014) 104 [arXiv:1401.5041] [INSPIRE].

[45] ATLAS collaboration, Measurements of Higgs boson production and couplings in the four-lepton channel in pp collisions at center-of-mass energies of 7 and 8 Te $V$ with the ATLAS detector, Phys. Rev. D 91 (2015) 012006 [arXiv:1408.5191] [INSPIRE].

[46] CMS collaboration, Measurement of the properties of a Higgs boson in the four-lepton final state, Phys. Rev. D 89 (2014) 092007 [arXiv:1312.5353] [INSPIRE].

[47] CMS collaboration, Precise determination of the mass of the Higgs boson and tests of compatibility of its couplings with the Standard Model predictions using proton collisions at 7 and 8 TeV, Eur. Phys. J. C 75 (2015) 212 [arXiv:1412.8662] [InSPIRE].

[48] ATLAS collaboration, Observation and measurement of Higgs boson decays to $W W^{*}$ with the ATLAS detector, Phys. Rev. D 92 (2015) 012006 [arXiv:1412.2641] [INSPIRE].

[49] ATLAS collaboration, Study of $(W / Z) H$ production and Higgs boson couplings using $H \rightarrow W W^{*}$ decays with the ATLAS detector, JHEP 08 (2015) 137 [arXiv:1506.06641] [INSPIRE].

[50] CMS collaboration, Measurement of Higgs boson production and properties in the $W W$ decay channel with leptonic final states, JHEP 01 (2014) 096 [arXiv: 1312.1129] [INSPIRE].

[51] ATLAS collaboration, Search for the $b \bar{b}$ decay of the Standard Model Higgs boson in associated $(W / Z) H$ production with the ATLAS detector, JHEP 01 (2015) 069 [arXiv:1409.6212] [INSPIRE].

[52] ATLAS collaboration, Search for the Standard Model Higgs boson produced in association

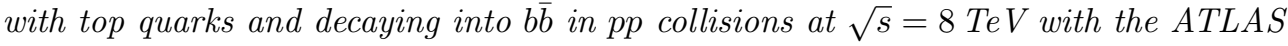
detector, Eur. Phys. J. C 75 (2015) 349 [arXiv:1503.05066] [InSPIRE].

[53] CMS collaboration, Search for the Standard Model Higgs boson produced in association with a $W$ or a $Z$ boson and decaying to bottom quarks, Phys. Rev. D 89 (2014) 012003 [arXiv:1310.3687] [INSPIRE].

[54] CMS collaboration, Search for the associated production of the Higgs boson with a top-quark pair, JHEP 09 (2014) 087 [Erratum ibid. 10 (2014) 106] [arXiv:1408.1682] [INSPIRE].

[55] CDF collaboration, T. Aaltonen et al., Combination of searches for the Higgs boson using the full CDF data set, Phys. Rev. D 88 (2013) 052013 [arXiv:1301.6668] [InSPIRE].

[56] D0 collaboration, V.M. Abazov et al., Combined search for the Higgs boson with the D0 experiment, Phys. Rev. D 88 (2013) 052011 [arXiv: 1303.0823] [INSPIRE]. 
[57] LHC Higgs Cross section Working Group collaboration, J.R. Andersen et al., Handbook of LHC Higgs cross sections: 3. Higgs properties, arXiv:1307.1347 [INSPIRE].

[58] R. Contino, M. Ghezzi, C. Grojean, M. Mühlleitner and M. Spira, eHDECAY: an implementation of the Higgs effective Lagrangian into HDECAY, Comput. Phys. Commun. 185 (2014) 3412 [arXiv: 1403.3381] [INSPIRE].

[59] R. Contino, C. Grojean, M. Moretti, F. Piccinini and R. Rattazzi, Strong double Higgs production at the LHC, JHEP 05 (2010) 089 [arXiv:1002.1011] [INSPIRE].

[60] G.F. Giudice, C. Grojean, A. Pomarol and R. Rattazzi, The strongly-interacting light Higgs, JHEP 06 (2007) 045 [hep-ph/0703164] [INSPIRE].

[61] A. Azatov, R. Contino and J. Galloway, Model-independent bounds on a light Higgs, JHEP 04 (2012) 127 [Erratum ibid. 04 (2013) 140] [arXiv:1202.3415] [INSPIRE].

[62] R. Contino, M. Ghezzi, C. Grojean, M. Muhlleitner and M. Spira, Effective Lagrangian for a light Higgs-like scalar, JHEP 07 (2013) 035 [arXiv:1303.3876] [INSPIRE].

[63] R. Barbieri, B. Bellazzini, V.S. Rychkov and A. Varagnolo, The Higgs boson from an extended symmetry, Phys. Rev. D 76 (2007) 115008 [arXiv: 0706. 0432] [INSPIRE].

[64] C. Grojean, W. Skiba and J. Terning, Disguising the oblique parameters, Phys. Rev. D 73 (2006) 075008 [hep-ph/0602154] [INSPIRE].

[65] A. Azatov, R. Contino, A. Di Iura and J. Galloway, New prospects for Higgs compositeness in $h \rightarrow Z \gamma$, Phys. Rev. D 88 (2013) 075019 [arXiv: 1308.2676] [INSPIRE].

[66] A. Pich, I. Rosell and J.J. Sanz-Cillero, Viability of strongly-coupled scenarios with a light Higgs-like boson, Phys. Rev. Lett. 110 (2013) 181801 [arXiv:1212.6769] [INSPIRE].

[67] A. Pich, I. Rosell and J.J. Sanz-Cillero, Oblique $S$ and $T$ constraints on electroweak strongly-coupled models with a light Higgs, JHEP 01 (2014) 157 [arXiv:1310.3121] [INSPIRE].

[68] ATLAS and CMS collaborations, Measurements of the Higgs boson production and decay rates and constraints on its couplings from a combined ATLAS and CMS analysis of the LHC pp collision data at $\sqrt{s}=7$ and 8 TeV, JHEP 08 (2016) 045 [arXiv:1606.02266] [INSPIRE].

[69] A. Falkowski, F. Riva and A. Urbano, Higgs at last, JHEP 11 (2013) 111 [arXiv: 1303.1812] [INSPIRE].

[70] J. Ellis and T. You, Updated global analysis of Higgs couplings, JHEP 06 (2013) 103 [arXiv:1303.3879] [INSPIRE].

[71] A. Djouadi and G. Moreau, The couplings of the Higgs boson and its CP properties from fits of the signal strengths and their ratios at the $7+8 \mathrm{TeV}$ LHC, Eur. Phys. J. C 73 (2013) 2512 [arXiv: 1303.6591] [INSPIRE].

[72] G. Bélanger, B. Dumont, U. Ellwanger, J.F. Gunion and S. Kraml, Global fit to Higgs signal strengths and couplings and implications for extended Higgs sectors, Phys. Rev. D 88 (2013) 075008 [arXiv:1306.2941] [inSPIRE].

[73] S. Choi, S. Jung and P. Ko, Implications of LHC data on $125 \mathrm{GeV}$ Higgs-like boson for the Standard Model and its various extensions, JHEP 10 (2013) 225 [arXiv:1307.3948] [INSPIRE]. 
[74] P. Bechtle, S. Heinemeyer, O. Stål, T. Stefaniak and G. Weiglein, Probing the Standard Model with Higgs signal rates from the Tevatron, the LHC and a future ILC, JHEP 11 (2014) 039 [arXiv: 1403.1582] [inSPIRE].

[75] J. Bergstrom and S. Riad, Bayesian model comparison of Higgs couplings, Phys. Rev. D 91 (2015) 075008 [arXiv:1411.4876] [inSPIRE].

[76] T. Corbett, O.J.P. Eboli, D. Goncalves, J. Gonzalez-Fraile, T. Plehn and M. Rauch, The Higgs legacy of the LHC run I, JHEP 08 (2015) 156 [arXiv: 1505.05516] [INSPIRE].

[77] TLEP Design Study Working Group collaboration, M. Bicer et al., First look at the physics case of TLEP, JHEP 01 (2014) 164 [arXiv:1308.6176] [INSPIRE].

[78] T. Barklow et al., ILC operating scenarios, arXiv:1506.07830 [INSPIRE].

[79] K. Fujii et al., Physics case for the International Linear Collider, arXiv:1506.05992 [INSPIRE].

[80] CEPC-SPPC Study GRoup collaboration, CEPC-SPPC preliminary conceptual design report. 1. Physics and detector, (2015) [INSPIRE].

[81] CEPC-SPPC Study GRoup collaboration, CEPC-SPPC preliminary conceptual design report. 2. Accelerator, (2015) [INSPIRE].

[82] CMS collaboration, Projected performance of an upgraded CMS detector at the LHC and HL-LHC: contribution to the Snowmass process, in Community Summer Study 2013:

Snowmass on the Mississippi (CSS2013), Minneapolis MN U.S.A. July 29-August 62013 [arXiv: 1307.7135] [INSPIRE].

[83] ATLAS collaboration, Projections for measurements of Higgs boson cross sections, branching ratios and coupling parameters with the ATLAS detector at a HL-LHC, ATL-PHYS-PUB-2013-014, CERN, Geneva Switzerland (2013).

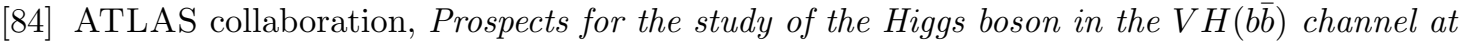
HL-LHC, ATL-PHYS-PUB-2014-011, CERN, Geneva Switzerland (2014).

[85] ATLAS collaboration, Projections for measurements of Higgs boson signal strengths and coupling parameters with the ATLAS detector at a HL-LHC, ATL-PHYS-PUB-2014-016, CERN, Geneva Switzerland (2014).

[86] J. Erler, S. Heinemeyer, W. Hollik, G. Weiglein and P.M. Zerwas, Physics impact of GigaZ, Phys. Lett. B 486 (2000) 125 [hep-ph/0005024] [INSPIRE].

[87] A. Freitas et al., Exploring quantum physics at the ILC, in Community Summer Study 2013: Snowmass on the Mississippi (CSS2013), Minneapolis MN U.S.A. July 29-August 62013 [arXiv: 1307.3962] [INSPIRE].

[88] J. Fan, M. Reece and L.-T. Wang, Possible futures of electroweak precision: ILC, FCC-ee and CEPC, JHEP 09 (2015) 196 [arXiv:1411.1054] [INSPIRE].

[89] S.-F. Ge, H.-J. He and R.-Q. Xiao, Probing new physics scales from Higgs and electroweak observables at $e^{+} e^{-}$Higgs factory, JHEP 10 (2016) 007 [arXiv: 1603.03385] [INSPIRE].

[90] P. Azzi, Progress in FCC-ee experimental studies, talk given at the FCC Week, Rome Italy (2016).

[91] S. Dawson et al., Working group report: Higgs boson, in Community Summer Study 2013: Snowmass on the Mississippi (CSS2013), Minneapolis MN U.S.A. July 29-August 62013 [arXiv: 1310.8361$]$ [INSPIRE]. 
[92] M. Baak et al., Working group report: precision study of electroweak interactions, in Community Summer Study 2013: Snowmass on the Mississippi (CSS2013), Minneapolis MN U.S.A. July 29-August 62013 [arXiv:1310.6708] [INSPIRE].

[93] A. Freitas, Electroweak precision tests in the LHC era and Z-decay form factors at two-loop level, in Proceedings, $12^{\text {th }}$ DESY Workshop on Elementary Particle Physics: loops and legs in quantum field theory (LL2014), (2014) [PoS(LL2014) 050] [arXiv:1406.6980] [INSPIRE].

[94] A. Freitas, Numerical multi-loop integrals and applications, Prog. Part. Nucl. Phys. 90 (2016) 201 [arXiv: 1604.00406] [INSPIRE].

[95] D.M. Asner et al., Physics at BES-III, Int. J. Mod. Phys. A 24 (2009) S1 [arXiv:0809.1869] [INSPIRE].

[96] V. Lubicz, private communication.

[97] A. Andreazza et al., What next: white paper of the INFN-CSN1, Frascati Phys. Ser. 60 (2015) 1 [INSPIRE].

[98] M. Beneke, A quark mass definition adequate for threshold problems, Phys. Lett. B 434 (1998) 115 [hep-ph/9804241] [INSPIRE].

[99] A.H. Hoang and T. Teubner, Top quark pair production close to threshold: top mass, width and momentum distribution, Phys. Rev. D 60 (1999) 114027 [hep-ph/9904468] [InSPIRE].

[100] M. Beneke, Y. Kiyo, P. Marquard, A. Penin, J. Piclum and M. Steinhauser, Next-to-next-to-next-to-leading order QCD prediction for the top antitop $S$-wave pair production cross section near threshold in $e^{+} e^{-}$annihilation, Phys. Rev. Lett. 115 (2015) 192001 [arXiv: 1506.06864] [INSPIRE].

[101] M. Beneke, A. Maier, J. Piclum and T. Rauh, Higgs effects in top anti-top production near threshold in $e^{+} e^{-}$annihilation, Nucl. Phys. B 899 (2015) 180 [arXiv:1506.06865] [INSPIRE].

[102] P. Marquard, A.V. Smirnov, V.A. Smirnov and M. Steinhauser, Quark mass relations to four-loop order in perturbative QCD, Phys. Rev. Lett. 114 (2015) 142002 [arXiv: 1502.01030] [INSPIRE].

[103] J. de Blas et al., Electroweak precision constraints at present and future colliders, arXiv: 1611.05354 [INSPIRE]. 